\title{
Dopamine Replacement Therapy Does Not Restore the Full Spectrum of Normal Pallidal Activity in the 1-Methyl-4- Phenyl-1,2,3,6-Tetra-Hydropyridine Primate Model of Parkinsonism
}

\author{
Gali Heimer, ${ }^{1,4}$ Michal Rivlin-Etzion, ${ }^{1,2}$ Izhar Bar-Gad, ${ }^{5}$ Joshua A. Goldberg, ${ }^{6}$ Suzanne N. Haber, ${ }^{7}$ and Hagai Bergman ${ }^{1,2,3}$ \\ ${ }^{1}$ Department of Physiology, ${ }^{2}$ Interdisciplinary Center for Neural Computation, and ${ }^{3}$ Eric Roland Center for Neurodegenerative Diseases, The Hebrew \\ University-Hadassah Medical School, Jerusalem, Israel, 91120, ${ }^{4}$ Department of Pediatrics, Hadassah-Hebrew University Medical Center, Jerusalem, Israel, \\ 91120, ${ }^{5}$ Gonda Brain Research Center, Bar-Ilan University, Ramat-Gan, Israel, 52900, ${ }^{6}$ Department of Biology, University of Texas at San Antonio, San \\ Antonio, Texas 78249, and 7Department of Pharmacology and Physiology, University of Rochester, Rochester, New York 14642
}

Current physiological studies emphasize the role of neuronal oscillations and synchronization in the pathophysiology of Parkinson's disease; however, little is known about their specific roles in the neuronal substrate of dopamine replacement therapy (DRT). We investigated oscillatory activity and correlations throughout the different states of levodopa-naive parkinsonism as well as "Off-On" and dyskinetic states of DRT in the external globus pallidum (GPe) of tremulous (vervet) and rigid-akinetic (macaque) monkeys and in the internal globus pallidum (GPi) of the vervet monkey. We found that, although oscillatory activity of cells and interneuronal correlation in both pallidal segments increases after induction of parkinsonism with 1-methyl-4-phenyl-1,2,3,6-tetra-hydropyridine (MPTP) and decreases in response to DRT, important differences exist between the two pallidal segments. In the GPi, the fraction of oscillatory cells and relative power of oscillations were significantly higher than in the GPe, and the dominant frequency was within the range of 7.5-13.5 $\mathrm{Hz}$ compared with a range of $4.5-7.5 \mathrm{~Hz}$ within the GPe. The interneuronal correlations were mostly oscillatory in the GPi, whereas at least half are non-oscillatory in the GPe. We demonstrate that the tremor characteristics after exposure to DRT do not resemble those of the normal or the levodopa-naive state. Moreover, although DRT reverses the MPTP-induced neuronal changes (rate, pattern, and pairwise correlations), the balance between GPe and GPi fails to restore. We therefore suggest that this imbalance reflects additional abnormal organization of the basal ganglia networks in response to dopamine replacement and may constitute the physiological substrate of the limitations and side effects of chronic DRT.

Key words: Parkinson's disease; basal ganglia; MPTP; tremor; cross-correlations; levodopa

\section{Introduction}

Early physiological studies of parkinsonian 1-methyl-4-phenyl1,2,3,6-tetra-hydropyridine (MPTP)-treated monkeys reported changes in the discharge rate within the external globus pallidum (GPe), internal globus pallidum (GPi) (Miller and DeLong, 1987; Filion and Tremblay, 1991), and the subthalamic nucleus (STN) (Bergman et al., 1994). Subsequent findings showed that inactivation of STN and GPi could improve the motor symptoms in parkinsonian animals (Bergman et al., 1990; Aziz et al., 1991) and human patients (Lang et al., 1997; Kumar et al., 2000; Krack et al.,

\footnotetext{
Received May 2, 2005; revised June 9, 2006; accepted June 10, 2006

This work was supported in part by a Center of Excellence Grant from the Israel Science Foundation and the "Fighting against Parkinson" Foundation of the Hebrew University-Netherlands Association (H.B.) and National Institutes of Health Grant MH 63324 (S.N.H.). V. Sharkansky provided technical support. We thank G. Goelman (Hadassah Hospital, Jerusalem, Israel) for assistance with the MRI imaging. We thank E. Vaadia for critical reading and fruitful discussions.

Correspondence should be addressed to Gali Heimer, Department of Physiology, The Hebrew University-Hadassah Medical School, P.0. Box 12272, Jerusalem, Israel 91120. E-mail: galih@md.huji.ac.il. D0I:10.1523/JNEUROSCI.5140-05.2006

Copyright $\odot 2006$ Society for Neuroscience $\quad$ 0270-6474/06/268101-14\$15.00/0
}

2003; Walter and Vitek, 2004). Finally, reversed trends of pallidal discharge rates in response to dopamine replacement therapy (DRT) have been reported in both human patients (Hutchinson et al., 1997a; Merello et al., 1999; Levy et al., 2001) and primates (Filion et al., 1991; Papa et al., 1999; Heimer et al., 2002).

These findings contributed to the formulation and the popularity of the rate model of the basal ganglia (Albin et al., 1989; DeLong, 1990). Nevertheless, subsequent studies have challenged the basic tenets of this model. Several studies have failed to find the expected significant changes of firing rates in the pallidum (Boraud et al., 1998; Raz et al., 2000), thalamus (Pessiglione et al., 2005), or motor cortical areas (Doudet et al., 1990; Watts and Mandir, 1992; Goldberg et al., 2002) of MPTP monkeys. Similarly, biochemical and metabolic studies indicate that GPe activity does not change in parkinsonism (Levy et al., 1997). Moreover, the rate model fails to explain the success of inactivation of the STN and GPi in the treatment of dyskinesia (Marsden and Obeso, 1994).

The inconsistencies with the rate model have brought more attention to the potential role of other aspects of neuronal activity 
such as firing patterns (Boraud et al., 2001; Wichmann and Soares, 2006) and neuronal synchronization (Bergman et al., 1998 ) in the pathophysiology of Parkinson's disease (PD). Recent studies have reported an increase in both oscillatory activity and correlation of pallidal cells in MPTP primates (Nini et al., 1995; Raz et al., 2000) and parkinsonian patients (Hurtado et al., 1999; Levy et al., 2000). Although abnormal pallidal synchronization has been shown to decrease in response to DRT (Heimer et al., 2002), detailed studies of pattern and synchronization during different DRT stages are still lacking. Moreover, recent human studies (Levy et al., 2002) have only found oscillatory neuronal correlation in tremulous patients, which leads to the question of whether the increased neuronal synchronization is not merely a byproduct of the tremor or of the activity of independent neural oscillators with similar frequencies.

Human studies are limited by constraints of recording duration, selected anatomical targets, and the clinical state of the patients (e.g., most operated patients have already developed dyskinesia). In this study, we combined multielectrode recordings in the pallidum of control and MPTP-treated monkeys with a newly improved tool for spectral analysis of spike trains (Rivlin-Etzion et al., 2006). Using these tools, we investigated the role of pallidal oscillatory and non-oscillatory correlation throughout the different clinical states of MPTP-induced parkinsonism and DRT (levodopa-naive parkinsonian state and optimal and dyskinesiainducing DRT states).

\section{Materials and Methods}

Animals and behavioral paradigm. Two monkeys, a vervet (African green monkey, Cercopithecus aethiops aethiops, female, weight of $3.8 \mathrm{~kg}$, monkey Q) and a rhesus (Macaca mulatta, female, weight of $5.7 \mathrm{~kg}$, monkey $\mathrm{R})$ were trained to perform a simple visuomotor task. The monkeys' health was monitored by a veterinarian, and their fluid consumption, diet, and weight were assessed daily. All procedures were in accordance with the National Institutes of Health Guide for the Care and Use of Laboratory Animals (1996) and with the Hebrew University guidelines for the use and care of laboratory animals in research, approved and supervised by the Institutional Committee for Animal Care and Use.

Surgical procedures. After training, an $18 \mathrm{~mm}$ Cilux recording chamber was attached to the skull over a trephine hole to allow access to the pallidum. The recording chamber was tilted $50^{\circ}$ laterally in the coronal plan, with its center targeted at the following stereotaxic coordinates (in $\mathrm{mm}$ ): monkey Q, anterior 13, lateral 7, height 3 (Contreras et al., 1981); monkey R, anterior 12, lateral 7, height 5 (Paxinos et al., 2000). The chamber coordinates were adjusted and then verified using magnetic resonance imaging (MRI) [BioSpec 4.7 tesla animal system (Bruker, Ettlingen, Germany), fast-spin echo sequence; effective echo time, $80 \mathrm{~ms}$; repetition time, $2.5 \mathrm{~s} ; 13$ coronal slices, $2 \mathrm{~mm}$ wide]. All surgical and MRI procedures were performed under deep general anesthesia.

Neural activity: recording and analysis. During recording sessions, the monkeys' heads were immobilized, and eight glass-coated tungsten microelectrodes (impedance of $0.3-1.2 \mathrm{M} \Omega$ at $1000 \mathrm{~Hz}$ ), confined within a cylindrical guide (1.65 mm inner diameter), were advanced separately (EPS; Alpha-Omega Engineering, Nazareth, Israel) into the pallidum.

Each electrode signal was amplified with a gain of 5000-20,000 and bandpass filtered with a $300-6000 \mathrm{~Hz}$ four-pole Butterworth filter (MCP +2.8; Alpha-Omega Engineering). This electrical activity was sorted and classified on-line using a template-matching algorithm (MSD 3.21; Alpha-Omega Engineering). The sampling rate of spike detection pulses and behavioral events was $12 \mathrm{kHz}$ (AlphaMap 5.0; Alpha-Omega Engineering). In the vervet monkey $\mathrm{Q}$, we also recorded the continuous analog output of the electrodes, which was sampled at $24 \mathrm{kHz}$.

Entry into the pallidum (the functional lateral border of the GPe) was easily recognized in our penetrations because of the considerably different firing rate, pattern, and spike shape of pallidal versus striatal neurons. The classification of each recorded cell as belonging to either the external or internal pallidum was determined by several criteria: the depth of the electrode [depth from the striatal-pallidal functional border of all GPe and GPi cells included in the study was $0.48 \pm 0.39$ and $2.54 \pm 0.82 \mathrm{~mm}$ (mean $\pm \mathrm{SD}$, respectively)], other anatomical/physiological structures identified along the electrode trajectory (e.g., border cells), and the firing pattern of the cell (which only served as a criterion in the normal state). If the classification as GPe or GPi was in doubt, the unit was excluded from the analysis. In monkey R, no GPi cells corresponded to all of the above criteria and we report only on the GPe activity of this monkey. Cells were selected for recording as a function of their signal-to-noise ratio and real-time assessment of their isolation quality. Only stable and well isolated (as judged by stable spike waveforms and off-line verification of stable firing rates) units were included in this study. The stable recording time was $24.5 \pm 10.6$ and $20.8 \pm 10.4$ min (mean \pm SD) in monkeys Q and R, respectively. Minimum stable recording time was 4 and $3.3 \mathrm{~min}$ for monkeys $\mathrm{Q}$ and $\mathrm{R}$, respectively. The total number of units that met the above criteria and were included in this study was as follows: 623 and 102 GPe units from monkeys Q and R, respectively, and 174 GPi units from monkey Q. We attempted to cover most of the area of the GPe and GPi. We estimated the spatial location of our recordings using the alignment of the $x-y$ coordinates of the chamber with the MR images and atlases (Contreras et al., 1981; Paxinos et al., 2000). Our penetrations span the stereotaxic range of $\mathrm{A} 10$ to $\mathrm{A} 15$ (GPe) and $\mathrm{A} 11$ to $\mathrm{A} 14(\mathrm{GPi})$ in monkey $\mathrm{Q}$ and $\mathrm{A} 9$ to $\mathrm{Al} 5(\mathrm{GPe})$ in monkey R.

We conducted quantitative analyses of firing rates and oscillatory firing patterns of all pallidal cells included in the study. Cross-correlations were calculated for pairs of neurons with overlapping periods of stable recording. Only neuronal pairs that were recorded by different electrodes were included to avoid possible artifacts in the cross-correlograms attributable to a shadowing effect of high discharge rates in cells recorded from the same electrode (Bar-Gad et al., 2001). Although the shadowing effect may be compensated for in neurons that exhibit a near Poissonian firing pattern (Bar-Gad et al., 2001), estimation of oscillatory correlations between neighboring units may be overbiased because of the shadowing effect. Statistical tests were accepted as significant at $p<0.01$ unless specified otherwise. The same threshold was applied when comparing the neural activity for both monkeys and for all clinical states.

Oscillatory activity of the cells was estimated using the power spectrum density of the spike trains (frequency resolution, $0.25 \mathrm{~Hz}$ ). To compensate for artifacts caused by the refractory period, we used the shuffling method (Rivlin-Etzion et al., 2006) in which the spectrum of the original spike train is divided by the mean spectrum of the locally $(T=\sim 175 \mathrm{~ms})$ shuffled $(n=20)$ spike trains. A confidence level $(p<0.01$, normalized to the total number of bins) for the compensated spectrum was constructed based on the high-frequency range of $270-300 \mathrm{~Hz}$, at which the spectrum was flat. A cell was considered oscillatory if its compensated spectrum contained at least two consecutive bins within the range of $4.5-30 \mathrm{~Hz}$ that crossed the $p=0.01$ confidence level.

For the analysis of neuronal correlation, we tested the null hypothesis of independent activity in both the time and frequency domains. We therefore searched for either significant peaks and troughs in the crosscorrelogram (a nonflat correlogram) or a peak in the cross-spectra (indicative of a significant periodic oscillatory correlation). The crosscorrelograms were calculated for $5000 \mathrm{~ms}$ offset, using $1 \mathrm{~ms}$ bins and recording edge corrections. Baseline firing rate and SD were estimated using the first and last $500 \mathrm{~ms}$ of the $\pm 5000 \mathrm{~ms}$ cross-correlogram. A peak

Table 1. Order of appearance of clinical symptoms in the MPTP monkeys

\begin{tabular}{lll}
\hline & \multicolumn{2}{l}{ Days after first MPTP injection } \\
\cline { 2 - 3 } Symptom & Monkey Q (vervet) & Monkey R (macaque) \\
\hline Lower limb dystonia & 3 & 3 \\
Flexed posture & 3 & 3 \\
Bradykinesia & 3 & 4 \\
Akinesia & 4 & 4 \\
Freezing & 4 & 5 \\
Rigidity & 5 & 7 \\
Tremor & 5 & 10 \\
\hline
\end{tabular}


A
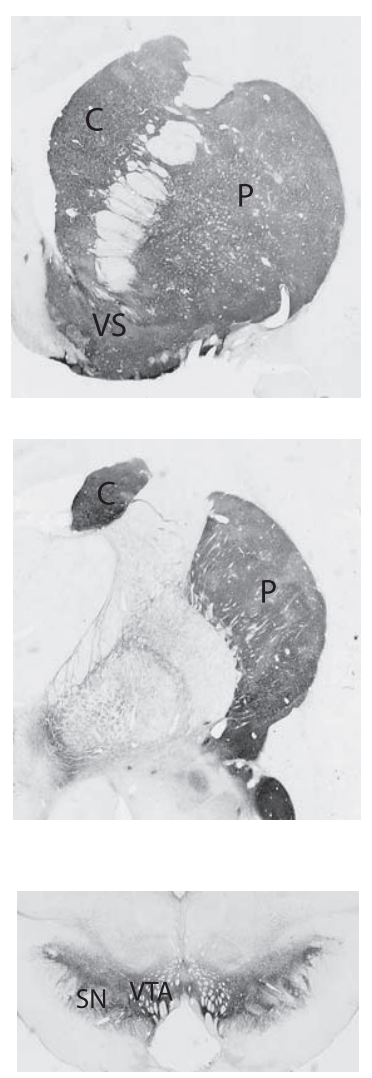

B
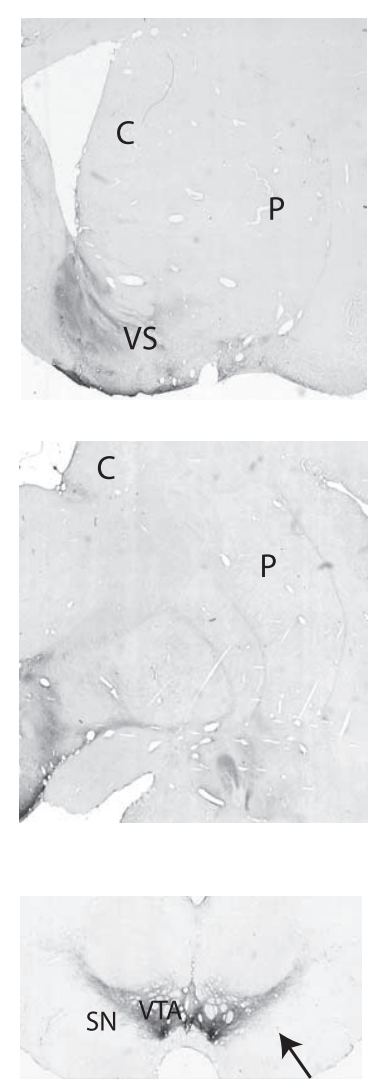

C
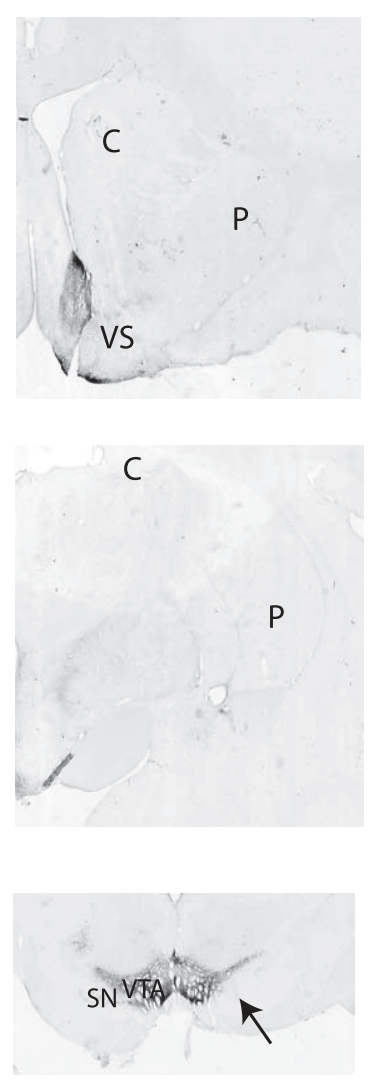

Figure 1. Photomicrographs of TH staining demonstrating the loss of dopaminergic substantia nigra pars compacta neurons in the MPTP-treated monkeys compared with a control animal. $\boldsymbol{A}$ was taken from a control normal macaque monkey; $\boldsymbol{B}$ and $\boldsymbol{C}$ are from the MPTP-treated monkeys $R$ (macaque) and $Q$ (vervet), respectively. The photomicrographs illustrate the levels of rostral striatum (row 1), central striatum (row 2), and midbrain (row 3). Note the lack of TH-positive staining throughout the striatum with the exception of the ventral striatum, particularly the shell region. TH-positive cells are selectively lost in the ventral tier (see arrows) but selectively spared in the ventral tegmental area. C, Caudate; $\mathrm{P}$, putamen; VS, ventral striatum; SN, substantia nigra; VTA, ventral tegmental area.

or trough was considered significant if it was made up of at least two consecutive bins that crossed the threshold of $p=0.01$ (normalized to the total number of bins) and was within an offset of $\pm 250 \mathrm{~ms}$ from 0 . The significance of the oscillatory correlations was assessed using the cross-spectral density of the spike trains. As in the analysis of single-cell oscillations, we used the shuffling method (Rivlin-Etzion et al., 2006). The cross-spectrum of the original spike trains was divided by the mean cross-spectrum of the globally shuffled $(n=20)$ spike trains. A confidence level ( $p<0.01$, normalized to the total number of bins) for the

compensated spectrum was constructed based on the high-frequency range of $270-300 \mathrm{~Hz}$, at which the spectrum was flat. A correlogram was considered to have significant periodic oscillations if its compensated spectrum contained at least two consecutive bins within the range of $4.5-30 \mathrm{~Hz}$ that crossed the $p=0.01$ confidence level. A noncorrelated pair was defined as a pair that had neither a significant peak in the crossspectra nor a significant peak or trough in the cross-correlogram. An oscillatory correlated pair was defined as a neuronal pair that had a significant peak in the cross-spectra. A nonoscillatory correlated pair was defined as a neuronal pair that only had a significant peak or trough in the cross-correlogram but did not have a significant peak in the cross-spectra.

Comparison between the mean neuronal firing rate throughout the different clinical states was done using the Student's $t$ test, with a significance of $p<0.01$ unless otherwise stated. In the analysis of rate changes in the continuously recorded cells, a significant change was defined as a decrease or increase of the firing rate of the cell by more than $10 \%$ of the baseline (before treatment) firing rate. The comparison of the fraction of neuronal oscillations and pairwise correlations in the different states was done using a $\chi^{2}$ test with a significance of $p<0.01$.

Tremor: recording and analysis. We used uniaxial accelerometers (8630C5; Kistler, Amherst, NY) to assess limb tremor. The analog output of the accelerometers was sampled at 712 and 521 $\mathrm{Hz}$ in monkeys $\mathrm{Q}$ and $\mathrm{R}$, respectively. Monkey $\mathrm{R}$ had one accelerometer fastened to its right hand (contralateral to the recorded hemisphere), and monkey Q had four accelerometers fastened to each of its four limbs. The accelerometers were attached distally on either the back of the hand or the foot. In the vervet monkey $Q$, we encountered many recording artifacts attributable to the strong tremor and collision of the monkey's limbs against solid surfaces, resulting in saturation of the recording apparatus and truncation of the data. We therefore only included accelerometer records that had a good signal-to-noise ratio and did not include such artifacts.

Visual inspection of the raw traces revealed that the tremor episodes tended to be short; hence, calculating the power spectra over long periods would lead to a misrepresentation of the spectral content of these episodes. We therefore cut the data into $10 \mathrm{~s}$ fragments and performed

Table 2. Accelerometer recording data

\begin{tabular}{|c|c|c|c|c|c|c|}
\hline & NOR & PNT & POT-on & POT-off & PDT-on & PDT-off \\
\hline \multicolumn{7}{|l|}{ Monkey Q (vervet) } \\
\hline Recording days & 10 & 12 & 6 & 6 & 9 & 9 \\
\hline Accelerometers $^{a}$ & 40 & 25 & 11 & 16 & 19 & 10 \\
\hline Total segments & 13,124 & 15,373 & 2442 & 2092 & 3979 & 1467 \\
\hline Tremulous segments (\%) & $4.5 \%$ & $41.0 \%$ & $50.9 \%$ & $43.2 \%$ & $42.3 \%$ & $45 \%$ \\
\hline Coherent pairs & $19.2 \%$ (5 of 26$)$ & $69.6 \%$ (39 of 56$)$ & $10.0 \%$ (3 of 30$)$ & $15.2 \%$ (5 of 33$)$ & $16.7 \%$ (5 of 30$)$ & $17.9 \%$ (5 of 28 ) \\
\hline \multicolumn{7}{|l|}{ Monkey R (macaque) } \\
\hline Recording days & 3 & 4 & & & 3 & 3 \\
\hline Accelerometers & 3 & 4 & & & 3 & 3 \\
\hline Total segments & 2038 & 1807 & & & 977 & 567 \\
\hline Tremulous segments (\%) & $1.3 \%$ & $4.5 \%$ & & & $14.8 \%$ & $0.7 \%$ \\
\hline
\end{tabular}

POT-on, On periods of parkinsonian monkey undergoing optimal DRT on a daily basis; POT-off, Off periods of parkinsonian monkey undergoing optimal DRT; PDT-on, On periods of parkinsonian monkey undergoing daily DRT after the development of dyskinesia; PDT-off, Off periods of parkinsonian monkey undergoing daily DRT treatment after the development of dyskinesia.

${ }^{a}$ Accelerometers refer to the number of accelerometer recordings within each state. In monkey $Q$, this number is higher than the number of recording days because of the multiple limb recordings and is lower than four times the number of recording days because of the discarding of sessions with artifacts. 
power spectra analysis for every segment with a 1024 bin fast Fourier transform, yielding a spectral resolution of 0.7 and $0.5 \mathrm{~Hz}$ in monkeys Q and $\mathrm{R}$, respectively. The SD of the spectrum noise was calculated from the tail of the spectrum $(55-200 \mathrm{~Hz})$. We considered all peaks that were over $7 \mathrm{SD}$ above a $p=0.01$ threshold to be significant (this combination of thresholds yielded the most similar results to visual judgment). Because of recording artifacts in low $(<3 \mathrm{~Hz})$ frequencies (presumed to reflect limb movements) and $\sim 30 \mathrm{~Hz}$ (presumed to result from the resonance frequency of the specific accelerometers used), we only included the peaks that were between the frequencies of 3.5 and 28 $\mathrm{Hz}$. If a segment had at least one significant peak within that range, it was considered tremulous. We calculated for each limb and each state the percentage of all the tremulous segments and the frequency distribution of the tremor frequencies of all the identified peaks.

In monkey $\mathrm{Q}$, the coherence between limb movements was calculated on the data of the simultaneously recorded accelerometers. For each pair of simultaneously recorded accelerometers, a coherence function was calculated for the same frequency range applied in the tremor analysis, and significant peaks were searched for in the range of 3.5-28 Hz. We checked the mean of the coherence function between 25 and $30 \mathrm{~Hz}$ as an indicator of the noise level of the coherence function and excluded the functions with mean noise $>0.055$ (a threshold chosen according to visual inspection of the data). In addition, we excluded the coherence function if the two accelerometers had an unequivocal noise artifact in the same frequency. We first used a standard significance criterion for the coherence function (Bloomfield, 1976; Brillinger, 1981):

$$
1-(1-a)^{\frac{1}{L-1}},
$$

where $a$ is the level of confidence (here $a=$ 0.999 ), and $L$ is the number of windows used in the calculation (length of the data divided by the window size, which in our case was 4096). We then applied a second threshold and considered as significant only those functions in which the integral between the two points in which the function crossed the significance line exceeded 0.04 (a threshold chosen according to visual inspection of the data). The overall coherence for each state was calculated as the percentage of accelerometer pairs that had at least one significant peak in their coherence function of the total number of possible pairs.

MPTP and dopamine replacement therapy. Parkinsonism was induced by five intramuscular injections of $0.4 \mathrm{mg} / \mathrm{kg}$ of the MPTP-HCl neurotoxin (Aldrich, Milwaukee, WI) over a period of $4 \mathrm{~d}$ (two injections on the first day). Both monkeys were clinically assessed on a regular basis using a modified primate clinical staging scale (Hoehn and Yahr, 1967; Imbert et al., 2000). In both monkeys, severe parkinsonism developed within $5 \mathrm{~d}$ from initiation of the four day MPTP treatment, and recordings were resumed $4 \mathrm{~d}$ after the last injection.

After $14 \mathrm{~d}$ of recordings in the parkinsonian state and $18 \mathrm{~d}$ after the last

A

$\mathrm{C}$

R
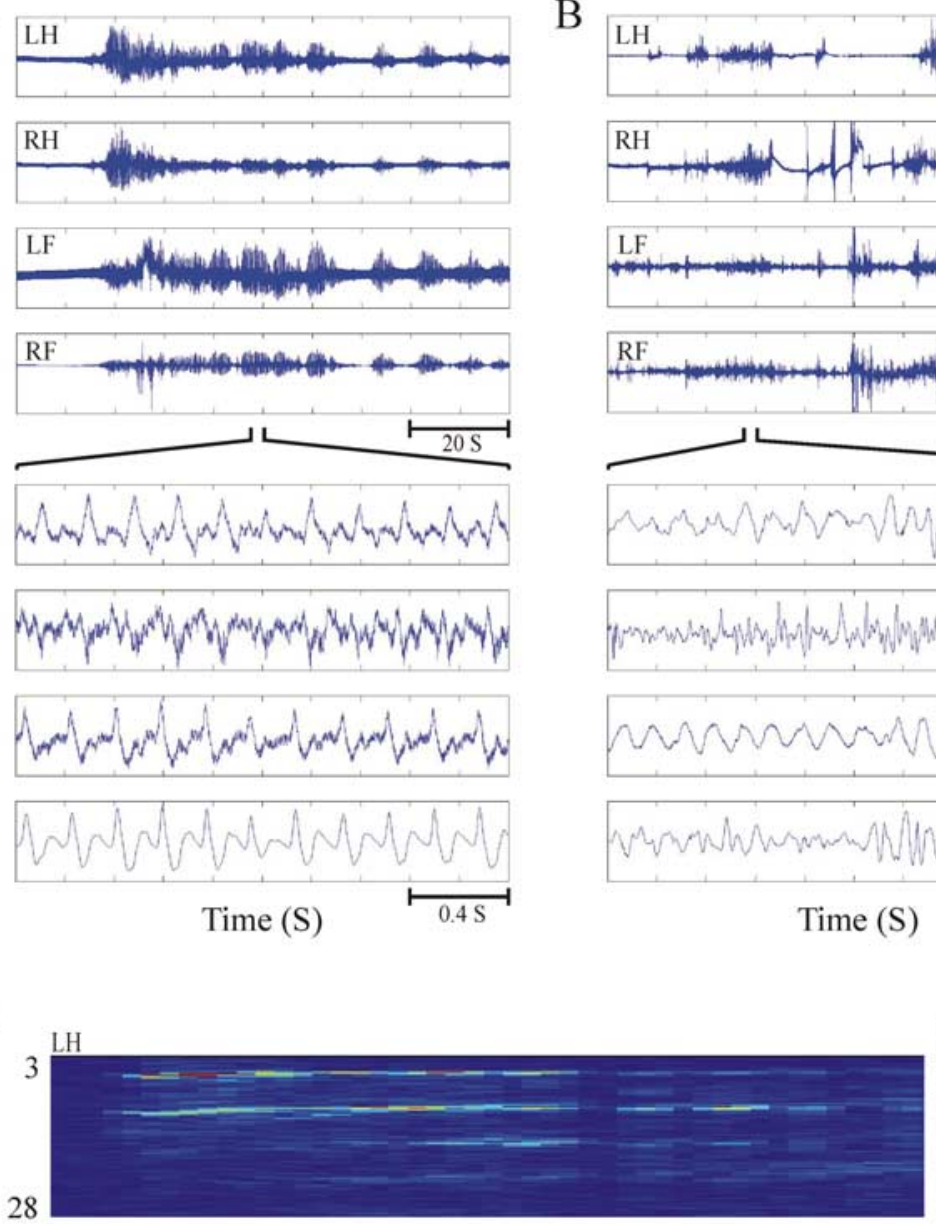

Relative power
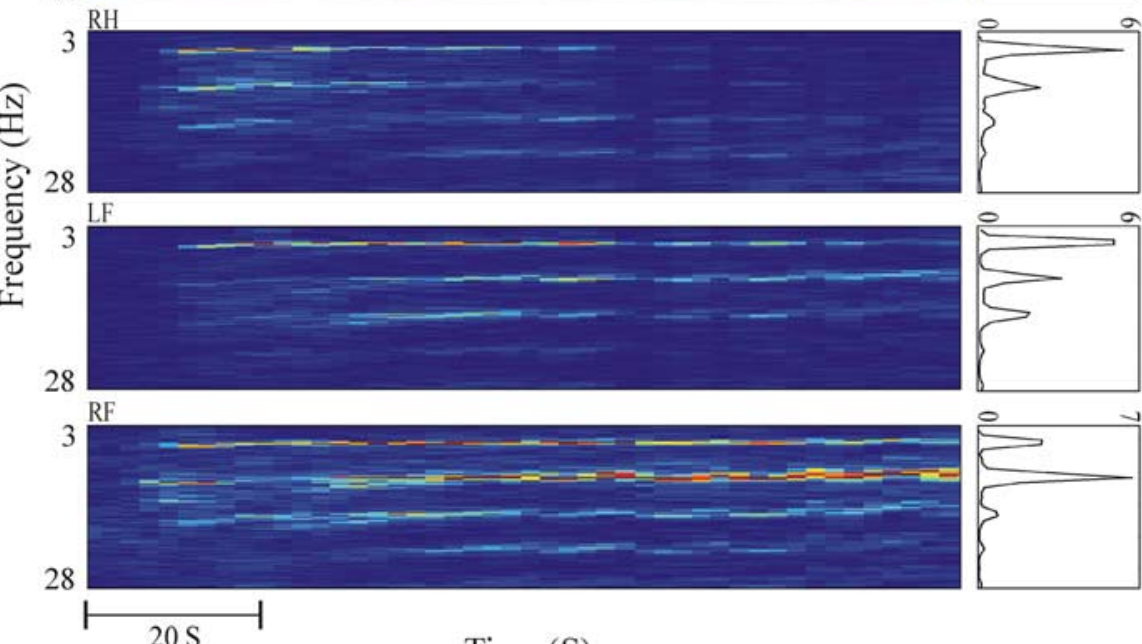

Time (S)

Figure 2. Examples of accelerometer recordings and analysis. $\boldsymbol{A}, \boldsymbol{B}$, Four simultaneous accelerometer recordings of limb tremor in monkey $Q$ in the levodopa-naive parkinsonian state $(\boldsymbol{A})$ and the 0 n period of optimally treated state $(\boldsymbol{B})$. The tremor is shown in two different timescales of 100 and $2 \mathrm{~s}$. The tremor in the levodopa-naive parkinsonian state is quite regular, and coherence between the limbs is evident in both between- and within-tremor episodes. In contrast, in the treatment state, the tremor traces are erratic and there is no apparent coherence between the limbs. $\boldsymbol{C}$, The spectrograms and power spectra of the same four simultaneously recorded accelerometer traces shown in the top panel of $\boldsymbol{A}$. The relative power ordinate is expressed as a logarithmic scale. LH, Left hand; RH, right hand; LF, left foot; RF, right foot.

MPTP injections (in both monkeys), we initiated dopamine replacement therapy on a daily basis. Starting doses for monkey Q were $0.5 \times 25 / 250$ mg of Dopicar [L-3,4-dihydroxyphenylalanine and carbidopa; Merck Sharp and Dohme, Haarlem, The Netherlands] in the morning and $5 \mathrm{mg}$ of Parlodel (Bromocryptine; Sandoz, Basel, Switzerland) divided equally 
A
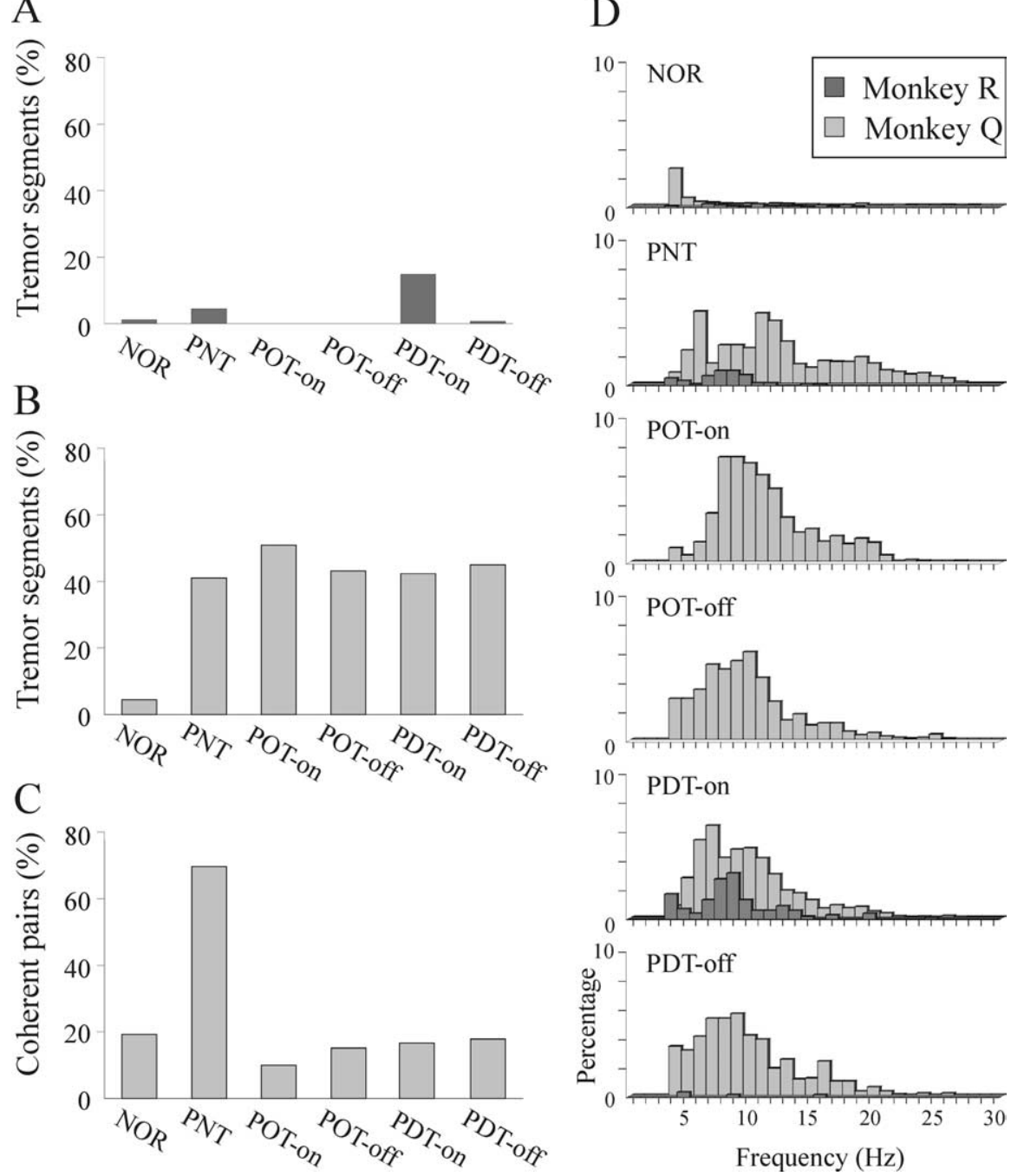

Figure 3. Results of the tremor analysis in both monkeys. $\boldsymbol{A}$ and $\boldsymbol{B}$ illustrate the percentage of segments that exhibited significant $3.5-28 \mathrm{~Hz}$ tremor, in monkeys $\mathrm{R}$ and $\mathrm{Q}$, respectively, throughout the clinical states. $C$ illustrates the percentage of coherence among all pairs of simultaneously recorded accelerometers in monkey $Q$ throughout the clinical states. Dillustrates the distribution of the tremor frequencies in both monkeys, from the normal state (top) to the dyskinetic Off state (bottom) in the same order as they appear in $\boldsymbol{A}-\boldsymbol{C}$. All significant $3.5-28 \mathrm{~Hz}$ tremor peaks (possibly more than 1 for a single segment) are shown. NOR, Normal state; PNT, parkinsonian levodopa-naive (not treated) monkey; POT-on, on periods of parkinsonian monkey undergoing optimal DRT on a daily basis; POT-off, Off periods of parkinsonian monkey undergoing optimal DRT; PDT-on, On periods of parkinsonian monkey undergoing daily DRT after the development of dyskinesia. PDT-off, Off periods of parkinsonian monkey undergoing daily DRT treatment after the development of dyskinesia.

between morning and evening. Starting doses for monkey R were $0.5 \times$ 25/250 mg of Dopicar and $5 \mathrm{mg}$ of Parlodel twice daily, in the morning and in the evening. The drugs were administered orally as crushed powder dissolved in liquid. The doses were slowly increased and adjusted to achieve optimal clinical response, and then the recordings were resumed. After a period of recording in the optimal treatment state, we gradually increased the doses until the development of dyskinesia and resumed the recordings once more. Maximal doses attained in monkey Q were $1.5 \times$ 25/250 mg of Dopicar with $2.5 \mathrm{mg}$ of Parlodel in the morning and $1 \times$ 25/250 mg of Dopicar with $2.5 \mathrm{mg}$ Parlodel in the evening. Maximal doses attained in monkey R were $1.75 \times 25 / 250 \mathrm{mg}$ of Dopicar with $5 \mathrm{mg}$ of Parlodel in the morning and $1.25 \times 25 / 250 \mathrm{mg}$ of Dopicar with $5 \mathrm{mg}$ of Parlodel in the evening.

The clinical state was assessed daily by human observation. During the recordings, "Off" periods were defined either as the periods before the morning dose or periods of over $5 \mathrm{~h}$ from the last dose providing there were clear symptoms of severe parkinsonism. The clinical definition of the "Off-On" transition was based on observation of limbs and tail movements, resumption of task performance, or appearance of involuntary dyskinetic movements (at the stage these had already developed). "On" periods were defined as the periods after the Off-On transition and up to $3 \mathrm{~h}$ from the administration of drugs. We use the following abbreviations for the clinical states: NOR refers to the normal state, PNT refers to the parkinsonian levodopa-naive ("no treatment") state, POT refers to the state of optimal treatment in the parkinsonian monkey, and PDT refers to the state of dyskinesia-inducing treatment in the parkinsonian monkey. When using the phrase "all parkinsonian states," we refer to the levodopa-naive state along with the Off states of both the optimal treatment (POT) and the dyskinetic treatment (PDT) periods. When referring to Off or On states without mentioning POT or PDT, we mean the Off or On states of both optimal and dyskinetic treatment. When referring to "all treatment states," we mean both the optimal and dyskinetic treatment states in monkey $\mathrm{Q}$ and the dyskinetic state in monkey $\mathrm{R}$.

In the recordings during the DRT state, we recorded each day for $10-30 \mathrm{~min}$ in the Off period before the morning dose. We then administered the medications while keeping the electrodes in position and subsequently resumed the recordings. In many cases, this protocol enabled recording of a given unit before, during, and after administration of DRT. In other cases, when units were lost during the oral administration of the drugs, it still enabled us to record the activity of other cells in the immediate vicinity of the cells studied before DRT. The protocol also made it possible in some instances to record the ongoing discharge changes of cells in response to the medication. In the data analysis, however, we only included the stable segments before and after these transients. Periods of complete cessation of GPi discharge after DRT were excluded from the analysis, because it was impossible to characterize the firing patterns and neuronal synchronization of cells that were virtually inactive. Because in monkey $\mathrm{R}$ we started using the above protocol only after the appearance of dyskinesia, the recordings of the optimal treatment state in this monkey did not match our Off/On criteria and were omitted from the study.

Histology. After the last recording session (98 d from last MPTP injection in monkey $\mathrm{Q}$ and $151 \mathrm{~d}$ in monkey $\mathrm{R}$ ), the monkeys were deeply anesthetized with a lethal dose of pentobarbital and perfused through the heart with saline, followed by a $4 \%$ paraformaldehyde fixative solution. Brains were removed and cryoprotected in increasing gradients of sucrose $(10,20$, and finally $30 \%)$. Adjacent serial sections of $50 \mu \mathrm{m}$, from both control animals and MPTP-treated animals, were processed for either a Nissl stain or immunocytochemistry for tyrosine hydroxylase (TH). Sections were incubated with antisera to TH (mouse anti-TH, 1:20,000; Eugene Tech, Allendale, $\mathrm{NJ})$ in $0.1 \mathrm{~m}$ phosphate buffer with $0.3 \%$ Triton $\mathrm{X}-100$ and $10 \%$ normal goat serum (Incstar, Stillwater, MN) for 4 nights at $4^{\circ} \mathrm{C}$ and further processed using the avidin-biotin method (rabbit Elite Vectastain ABC kit; Vector Laboratories, Burlingame, CA). Nissl histology was used to verify the recording location. However, the use of multiple-electrode recording, with a guide of outer diameter $2.2 \mathrm{~mm}$, does not enable exact reconstruction of all penetration tracks, and therefore we only verified the recording boundaries to be inside the 
pallidum. To estimate the dopaminergic fiber loss, the striatum was divided into three regions based on cortical inputs (limbic, associative, and motor) and analyzed for optical density of $\mathrm{TH}$-positive fibers (NIH Image version 1.63). Mean density measurements were corrected for background staining by subtracting the average density sampled from white matter areas in each section. To standardize intensity across scans, an autoradiographic $\left[{ }^{14} \mathrm{C}\right]$ microscale, multilevel reference strip (Amersham Biosciences, Arlington Heights, IL) was used to calibrate NIH Image.

\section{Results}

\section{Clinical states}

The first signs of parkinsonism appeared on the third day of the MPTP injections in both monkeys and continued to evolve over the following 2-7 d (Table 1). Many prolonged episodes of low-frequency distal tremor were observed in monkey Q (vervet), whereas the short-lived tremor episodes of monkey R (macaque) appeared mostly in response to agitation and involved mainly the axial and proximal muscles. Both monkeys remained in a stable condition of severe parkinsonism during all recording days in the parkinsonian levodopa-naive state.

Dopamine replacement therapy commenced $18 \mathrm{~d}$ after the last MPTP injection in both monkeys. The first response to therapy was seen after the third dose of medication in monkey $\mathrm{Q}$ and the fourth dose in monkey $\mathrm{R}$ ( $\sim 24 \mathrm{~h}$ after initiation of treatment). The clinical effects of DRT included regaining of ability to self-feed, an increase in amount and velocity of movements, straightening of posture, and also a resumption (although suboptimal) of performance of the behavioral paradigm. The effects of DRT did not include a reduction of clinically observed tremor (for similar observations in human patients, see Vidailhet et al., 1999). In the macaque monkey $\mathrm{R}$, which was relatively nontremulous in the parkinsonian levodopa-

A

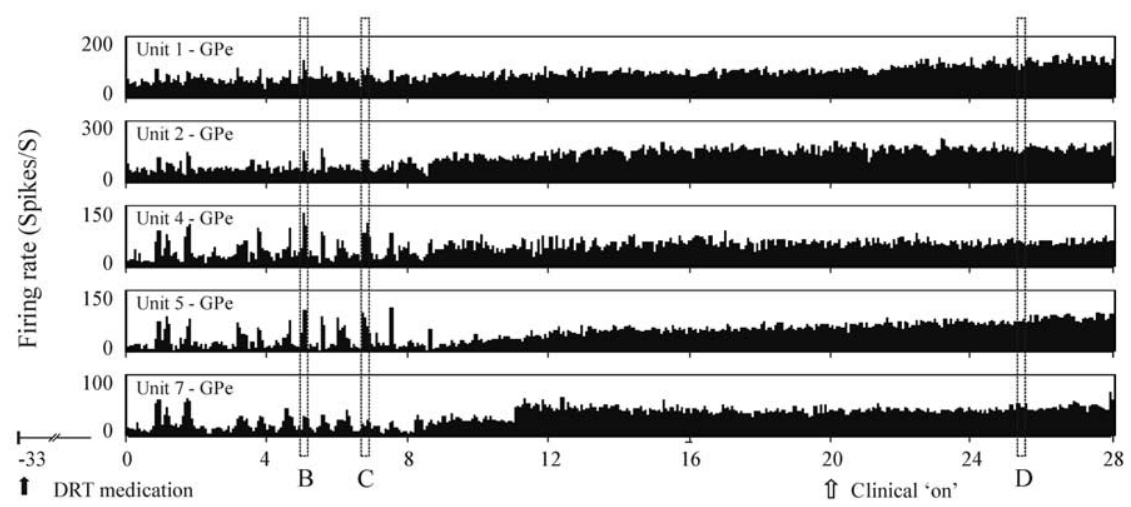

Time (Min)
B

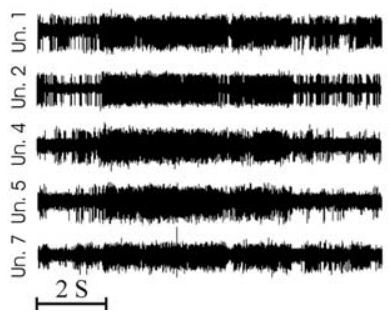

C

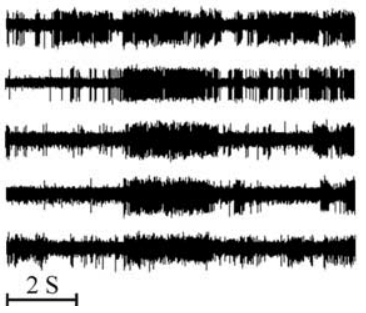

D

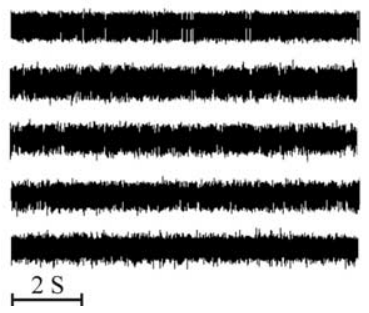

Figure 4. Discharge rates of five GPe neurons recorded simultaneously and continuously throughout the clinical Off-On transition in the dyskinetic treatment state (PDT). $\boldsymbol{A}$, Continuous 28 min recording illustrates changes in the neuronal discharge rates, pattern, and synchronization in response to DRT. Medication was administered orally 33 min before onset of recording (black arrow); clinical Off-On transition was noted after 20 min of recording (open arrow). $\boldsymbol{B}-\boldsymbol{D}$, Ten second traces of the raw analog recording of the same cells shown in $\boldsymbol{A}$. Note the repeated long (several seconds) synchronous bursts in the off period $(\boldsymbol{B}, \boldsymbol{C})$ that disappear in the consecutive On period (D).

naive state, the On state was accompanied by the appearance of tremor episodes. In the tremulous vervet monkey, there was an increase in apparent amplitude of the tremor rather than a change in its incidence. The first signs of peak-dose dyskinesia appeared after 6 and 7 weeks of daily DRT in monkeys Q and R, respectively. The dyskinesia manifested as overall hyperactivity, involuntary jerks of the limbs, torticolis, and episodes of circling.

Table 3. Pallidal firing rates, oscillatory activity, and interneuronal correlations

\begin{tabular}{|c|c|c|c|c|c|c|}
\hline & NOR & PNT & POT-on & POT-off & PDT-on & PDT-off \\
\hline \multicolumn{7}{|l|}{$\begin{array}{l}\text { A. Neuronal firing rates } \\
\text { (mean } \pm \text { SEM) }\end{array}$} \\
\hline GPe monkey R & $64.8 \pm 5.7(n=17)$ & $61.5 \pm 4.2(n=47)$ & & & $75.0 \pm 5.7(n=23)$ & $47.4 \pm 2.9(n=15)$ \\
\hline GPe monkey Q & $63.6 \pm 1.7(n=173)$ & $48.8 \pm 1.3(n=181)$ & $80.8 \pm 4.2(n=32)$ & $45.7 \pm 2.8(n=56)$ & $80.5 \pm 3.6(n=85)$ & $30.3 \pm 1.8(n=96)$ \\
\hline GPi monkey Q & $67.3 \pm 4.2(n=34)$ & $71.5 \pm 3.9(n=50)$ & $43.5 \pm 10.3(n=12)$ & $79.8 \pm 4.5(n=35)$ & $32.6 \pm 5.8(n=28)$ & $55.3 \pm 4.5(n=17)$ \\
\hline \multicolumn{7}{|c|}{ B. Percentage of oscillatory cells } \\
\hline GPe monkey R & $0 \%(0$ of 17$)$ & $44.7 \%$ (21 of 47$)$ & & & $17.4 \%$ (4 of 23 ) & $13.3 \%$ (2 of 15$)$ \\
\hline GPe monkey Q & $3.5 \%$ ( 6 of 173$)$ & $33.7 \%$ (61 of 181$)$ & $6.3 \%(2$ of 32$)$ & $12.5 \%(7$ of 56$)$ & $3.5 \%(3$ of 85$)$ & $14.6 \%$ (14 of 96$)$ \\
\hline Gpi monkey Q & $2.9 \%(1$ of 34$)$ & $82.0 \%(41$ of 50$)$ & $25.0 \%$ (3 of 12$)$ & $71.4 \%$ (25 of 35$)$ & $10.7 \%$ (3 of 28$)$ & $64.7 \%(11$ of 17$)$ \\
\hline \multicolumn{7}{|c|}{ C. Percentage of correlated pairs } \\
\hline GPe-GPe monkey R & $8.3 \%(1$ of 12$)$ & $32.3 \%(20$ of 62$)$ & & & $30.8 \%$ (8 of 26 ) & $70.0 \%$ (21 of 30$)$ \\
\hline GPe-GPe monkey Q & $16.2 \%$ (53 of 327 ) & $38.3 \%$ (164 of 428$)$ & $17.5 \%$ (7 of 40$)$ & $54.8 \%$ (57 of 104) & $21.3 \%$ (29 of 136$)$ & $67.9 \%$ (161 of 237$)$ \\
\hline GPi-GPi monkey Q & $16.3 \%$ (7 of 43 ) & $91.8 \%$ (56 of 61$)$ & $55.6 \%$ (5 of 9$)$ & $82.6 \%$ (19 of 23 ) & $22.7 \%$ (5 of 22$)$ & $85.0 \%(17$ of 20$)$ \\
\hline \multicolumn{7}{|c|}{$\begin{array}{l}\text { D. Percentage of oscillatory out } \\
\text { of all correlated pairs }\end{array}$} \\
\hline GPe-GPe monkey R & $0 \%(0$ of 1$)$ & $5.0 \%(1$ of 20$)$ & & & $0 \%(0$ of 8$)$ & $4.8 \%$ (1 of 21$)$ \\
\hline GPe-GPe monkey Q & $3.8 \%$ ( 2 of 53$)$ & $43.3 \%$ (71 of 164$)$ & $0 \%(0$ of 7$)$ & $14.0 \%(0$ of 7$)$ & $0 \%(0$ of 29$)$ & $5.6 \%(9$ of 161$)$ \\
\hline GPi-GPi monkey Q & $0 \%(0$ of 7$)$ & $100.0 \%$ (56 of 56$)$ & $100.0 \%$ (5 of 5 ) & $89.5 \%$ (17 of 19) & $0 \%(0$ of 5$)$ & $82.4 \%$ (14 of 17$)$ \\
\hline
\end{tabular}

Definitions are as in Table 1. 

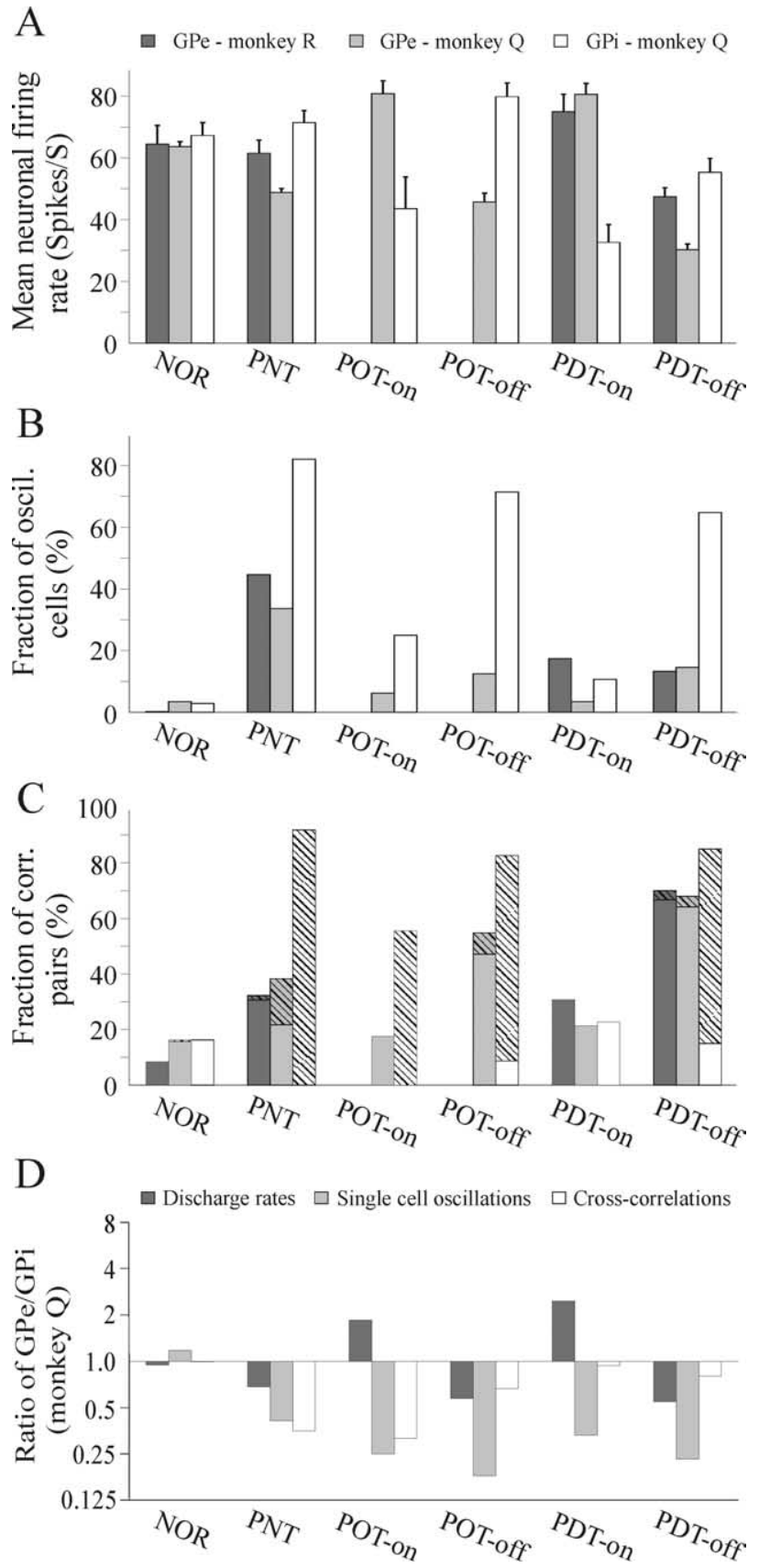

Figure 5. A-C, Summary of mean pallidal firing rates, fraction of oscillatory cells, and fraction of correlated neuronal pairs throughout the clinical states. $A, G$ Pe firing rates decrease in the dopaminedepleted states and increase in response to dopamine replacement. The opposite occurs in the neurons of the GPi. The error bars represent the SEM. Clinical state definitions are as in Figure 3 . B, Fraction of $4.5-30 \mathrm{~Hz}$ oscillatory cells is increased in both nuclei after induction of parkinsonism but is more pronounced in the GPi. During Off periods of treatment, oscillatory level in the GPi remains high, whereas in the GPe it is significantly lower than the levodopa-naive state. C, Neuronal non-oscillatory (filled bars) and oscillatory (striped bars) correlation in the pallidum. Neuronal correlation in the GPe is mainly non-oscillatory. It is increased in all parkinsonian states and reversed to near normal levels in response to dopamine replacement. Non-oscillatory correlation level is gradually increased from the levodopa-naive parkinsonian state throughout the Off states of treatment. In the GPi, neuronal correlation is mostly oscillatory and reaches higher levels than in the GPe. The correlation level decreases only partially in the 0 state of optimal treatment and profoundly in the dyskinetic 0 state. $D$, The balance of GPe/GPineuronal activity that exists in the normal state is disrupted after MPTP and fails to be restored by DRT. The GPe/GPi rate ratio decreases in the parkinsonian states and overshoots in response to DRT. In all parkinsonian and treated states, single-cell oscillatory activity is stronger within the GPi. Whereas in the PNT and POT states the correlated activity within the GPi is considerably stronger than within GPe, in the PDT states the GPe/GPi correlation ratio approximates 1 . The ordinate is given as a logarithmic scale.

\section{Histology}

In the control animals, there was dense TH immunoreactivity throughout the striatum (Fig. 1A). In contrast, both MPTPtreated animals had optical density measures close to 0 throughout the striatum, with the exception of the limbic region (Fig. $1 B, C)$. At the microscopic level, few fibers remained in the dorsal striatum. The two MPTP animals did not differ with respect to TH fiber loss. Cell loss in the midbrain was almost complete in the ventral tier of the substantia nigra pars compacta. However, there were a few remaining cells in both animals. As expected (Song and Haber, 2000), the cells in the ventral tegmental area of the midbrain dopamine system remained relatively spared.

\section{Accelerometers and tremor}

Table 2 summarizes the total number of accelerometer recording days and the final number of accelerometers and segments used in the analysis. In the vervet monkey Q, the final number of accelerometry recordings used for the analysis was relatively low compared with the number of recording days attributable to the discarding of sessions with artifacts. Figure 2 presents an example of raw data and spectrograms of simultaneous recordings of four accelerometers in the vervet monkey $\mathrm{Q}$ during the levodopanaive parkinsonian state (Fig. $2 A, C$ ) and the On state of optimal treatment (Fig. 2B).

In the normal state, the percentage of tremulous segments was relatively low in both monkeys (Fig. $3 A, B$ ). There was only a mild increase in the fraction of tremulous segments after MPTP in the parkinsonian macaque monkey $\mathrm{R}$, whereas in the vervet monkey $\mathrm{Q}$, it increased drastically to $\sim 40 \%$ of the segments. Throughout the optimal and dyskinetic treatment states in monkey Q, the percentage of tremulous segments remained high during both the Off and On periods. In monkey R, there was a clear increase in percentage of tremor in response to DRT in the dyskinetic state, reaching an even higher level than in the levodopa-naive parkinsonian state.

The level of coherence between pairs of simultaneously recorded accelerometers in monkey $\mathrm{Q}$ is shown in Figure 3C. Along with the increase in the fraction of tremor segments, the interlimb coherence level also increased dramatically in the levodopanaive parkinsonian state. Nevertheless, after introduction of DRT, the coherence level decreased to the same level as in the normal state despite the sustained high percentage of tremor episodes. The high interlimb coherence in the levodopa-naive parkinsonian monkey and its absence in the treated monkey are illustrated in Figure 2, A and $B$, respectively, both between discrete tremor episodes and in the tremor waveforms within the episodes. Moreover, in the treated state, there was irregularity not only between accelerometers but also within the trace of each limb itself. These differences in the characteristics of the tremor were also reflected in the incidence of the different tremor frequencies in the six clinical states (Fig. 3D). Although in the levodopa-naive parkinsonian state of the vervet monkey $\mathrm{Q}$ there were two distinct tremor peaks at $\sim 6$ and $11 \mathrm{~Hz}$ (Raz et al., 2000), the tremor frequencies in all treatment states were widely distributed.

\section{Neuronal firing rates}

An example of a continuous recording of five GPe units during the Off-On transition in the dyskinetic state is shown in Figure 4. The continuous recording displays the rate changes as well as modifications of discharge patterns in response to DRT. Consistent with previous reports, the GPe cells increased their firing rate drastically in response to the medication. In addition, whereas in the Off period the GPe cells discharged in a synchronized burst- 
Table 4. Changes in firing rates, oscillatory firing patterns, and synchronization in pallidal neurons continuously recorded over the Off-On transition

\begin{tabular}{|c|c|c|c|c|c|c|c|c|c|c|}
\hline \multirow{2}{*}{ A. Firing $r$} & \multicolumn{5}{|c|}{ Optimal treatment state } & \multicolumn{5}{|c|}{ Dyskinetic treatment state } \\
\hline & & & & & & & & & & \\
\hline & $n$ & Net change $^{a}$ & $\ln c^{b}$ & $\mathrm{NC} C^{b}$ & $\operatorname{Dec}^{b}$ & $n$ & Net change ${ }^{a}$ & $\ln c^{b}$ & $\mathrm{NC}{ }^{b}$ & $\operatorname{Dec}^{b}$ \\
\hline GPe & 12 & $\uparrow{ }^{*} 1.6(+36 \mathrm{spk} / \mathrm{s})$ & $\begin{array}{l}9 \\
(75 \%)\end{array}$ & $\begin{array}{l}3 \\
(25 \%)\end{array}$ & $\begin{array}{l}0 \\
(0 \%)\end{array}$ & 44 & $\uparrow{ }^{*} 2.2(+38 \mathrm{spk} / \mathrm{s})$ & $\begin{array}{l}37 \\
(84 \%)\end{array}$ & $\begin{array}{l}3 \\
(9 \%)\end{array}$ & $\begin{array}{l}4 \\
(7 \%)\end{array}$ \\
\hline GPi & 4 & $\downarrow{ }^{*} 1.2(-10 \mathrm{spk} / \mathrm{s})$ & $\begin{array}{l}1 \\
(25 \%)\end{array}$ & $\begin{array}{l}1 \\
(25 \%)\end{array}$ & $\begin{array}{l}2 \\
(50 \%)\end{array}$ & 4 & $\downarrow{ }^{*} 1.8(-27 \mathrm{spk} / \mathrm{s})$ & $\begin{array}{l}0 \\
(0 \%)\end{array}$ & $\begin{array}{l}0 \\
(0 \%)\end{array}$ & $\begin{array}{l}4 \\
(100 \%)\end{array}$ \\
\hline \multicolumn{11}{|c|}{ B. Oscillatory firing patternc } \\
\hline & $n$ & $\begin{array}{l}\text { Osc } \\
\downarrow \\
\text { NoOsc }\end{array}$ & $\begin{array}{l}\text { Osc } \\
\downarrow \\
\text { Osc }\end{array}$ & $\begin{array}{l}\text { NoOsc } \\
\downarrow \\
\text { NoOsc }\end{array}$ & $\begin{array}{l}\text { NoOsc } \\
\downarrow \\
\text { Osc }\end{array}$ & $n$ & $\begin{array}{l}\text { Osc } \\
\downarrow \\
\text { NoOsc }\end{array}$ & $\begin{array}{c}\text { Osc } \\
\downarrow \\
\text { Osc }\end{array}$ & $\begin{array}{l}\text { NoOsc } \\
\downarrow \\
\text { NoOsc }\end{array}$ & $\begin{array}{c}\text { NoOsc } \\
\downarrow \\
\text { Osc }\end{array}$ \\
\hline $\mathrm{GPe}$ & 12 & $17 \%$ & $8 \%$ & $75 \%$ & $0 \%$ & 44 & $12 \%$ & $2 \%$ & $84 \%$ & $2 \%$ \\
\hline GPi & 4 & $0 \%$ & $75 \%$ & $25 \%$ & $0 \%$ & 4 & $0 \%$ & $50 \%$ & $50 \%$ & $0 \%$ \\
\hline \multicolumn{11}{|c|}{ C. Pairwise neuronal synchronization ${ }^{d}$} \\
\hline & $n$ & $\begin{array}{l}\text { Cor } \\
\downarrow \\
\text { NoCor }\end{array}$ & $\begin{array}{c}\text { Cor } \\
\downarrow \\
\text { Cor }\end{array}$ & $\begin{array}{l}\text { NoCor } \\
\downarrow \\
\text { NoCor }\end{array}$ & $\begin{array}{l}\text { NoCor } \\
\downarrow \\
\text { Cor }\end{array}$ & $n$ & $\begin{array}{l}\text { Cor } \\
\downarrow \\
\text { NoCor }\end{array}$ & $\begin{array}{l}\text { Cor } \\
\downarrow \\
\text { Cor }\end{array}$ & $\begin{array}{l}\text { NoCor } \\
\downarrow \\
\text { NoCor }\end{array}$ & $\begin{array}{l}\text { NoCor } \\
\downarrow \\
\text { Cor }\end{array}$ \\
\hline GPe & 13 & $15 \%$ & $15 \%$ & $70 \%$ & $0 \%$ & 101 & $46 \%$ & $26 \%$ & $25 \%$ & $3 \%$ \\
\hline GPi & 5 & $40 \%$ & $40 \%$ & $20 \%$ & $0 \%$ & 2 & $100 \%$ & $0 \%$ & $0 \%$ & $0 \%$ \\
\hline
\end{tabular}

GPe continuously recorded cells are from both monkeys.

${ }^{a}$ The average change of firing rate for all continuously recorded cells. The change is expressed as the factor by which the average rate increased or decreased and by spikes per second (spk/s).

${ }^{b}$ The number of cells that decreased (Dec), increased (Inc), or did not change (NC) their discharge rate significantly after the medication. An increase or decrease of $>10 \%$ from the baseline rate of the cell was considered significant.

The fraction of cells that changed their discharge pattern from oscillatory (Osc) to non-oscillatory (NoOsc), remained oscillatory, remained non-oscillatory, or changed their pattern from non-oscillatory to oscillatory in response to the medication.

${ }^{d}$ The fraction of neuronal pairs that changed their correlation mode from correlated (Cor) to noncorrelated (NoCor), remained correlated, remained noncorrelated, or changed their mode from noncorrelated to correlated in response to the medication.

ing manner, under the influence of medication, this bursting activity subsided considerably.

Table $3 A$ summarizes the mean firing rates of all GPe and GPi cells recorded in each clinical state. After MPTP treatment, GPe firing rates decreased only slightly in the macaque monkey $\mathrm{R}$ but significantly in the vervet monkey Q (Fig. 5A). During treatment, the GPe rates in the Off states continued to decrease in both monkeys so that, in the PDT-Off state, the GPe firing rates were significantly lower than those in the normal state in monkey $\mathrm{R}$ as well. In response to DRT (On states), the GPe rates increased significantly compared with the Off state in both monkeys and exceeded the normal state rates. The increase in GPi rates after MPTP was not significant; however, the GPi rates in the POT-Off state increased further and were marginally $(p<0.05)$ significantly higher than in the normal state (Fig. $5 A$ ). In response to DRT, in both optimal and dyskinetic states, neuronal rates in the GPi decreased significantly and were also significantly lower than in the normal state. While in the normal state, the ratio of GPe to GPi mean neuronal firing rates approached 1; in all parkinsonian states, it decreased by nearly twofold (Fig. 5D). Conversely, in the optimal treatment On state, the mean GPe rates were almost twice the mean GPi rates, and this disparity was even larger in the dyskinetic On state. The results of the continuously (before and after the clinical influence of DRT) recorded neurons verify the population averages given above (Table $4 A$ ).

\section{Oscillatory activity of single neurons}

Figure 6 presents examples of raw analog traces, autocorrelation functions, power spectra, and spectrograms of oscillatory GPi (Fig. 6A,B) and GPe (Fig. 6C,D) cells during the levodopa-naive parkinsonian state (Fig. $6 \mathrm{~A}, C$ ) and Off state of optimal treatment (Fig. $6 B, D$ ). Table $3 B$ summarizes the percentage of all $4.5-30 \mathrm{~Hz}$ oscillatory GPe and GPi cells in each clinical state. Figure 7 depicts two examples of the complex and dynamic relationships between the pallidal neuronal oscillations and the tremor. It should be noted that, although this analysis was conducted in the levodopa-naive period when the tremor of different body segments is highly coherent (Fig. $3 C$ ), there is only a partial overlap in the times of oscillatory neuronal activity and tremor. In line with previous reports (Lemstra et al., 1999; Raz et al., 2000; Hurtado et al., 2005), these results support the hypothesis of dynamical functional connection between the basal ganglia networks involved in tremor generation and the skeleton-motor periphery. We therefore limited this paper to population level analysis of the tremor and the pallidal oscillations (see below).

After induction of parkinsonism, there was a significant increase in the percentage of oscillatory GPe cells in both monkeys (Fig. 5B). During Off states of the treatment phases, however, the fraction of oscillatory GPe cells was significantly lower than in the levodopa-naive parkinsonian state $(p<0.01$ for monkey $\mathrm{Q}$ and $p<0.05$ for monkey $\mathrm{R}$ ). Although there was a small decrease in the fraction of oscillatory cells in response to DRT in the GPe of the vervet monkey $Q$, it was not significant in the optimal treatment and significant only at $p<0.05$ in the dyskinetic treatment. In the GPe of monkey $\mathrm{R}$, there was no significant change in the fraction of oscillatory cells in response to DRT. The fraction of oscillatory GPe cells in the On states of treatment in both monkeys was not significantly different than in the normal state.

In the GPi of monkey Q, there was a vast increase in oscillatory activity after induction of parkinsonism (Fig. 5B). Nonetheless, in contrast to the GPe, the fraction of oscillatory GPi cells remained high in the Off periods of the treatment states and was not significantly different than in the levodopanaive parkinsonian state. Moreover, again in contrast to the $\mathrm{GPe}$, in the GPi, there was a significant decrease in the fraction of oscillatory cells in response to DRT in both the optimal and dyskinetic treatment. The fact that rate changes in the two pallidal segments went in opposite directions, whereas the changes in oscillatory activity were in the same direction, rules out the possibility that detection of oscillations was merely an artifact of the rate changes.

In all parkinsonian states, the fraction of oscillatory cells in the 
A
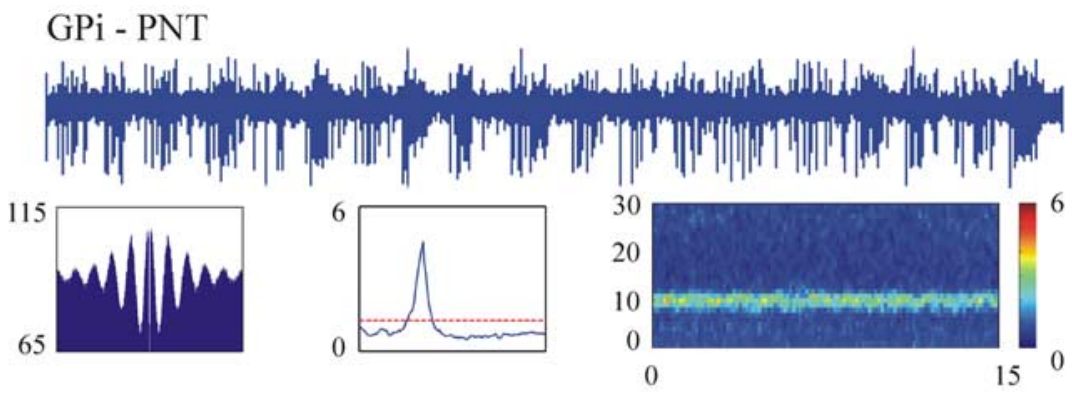

B GPi - POT-'off'
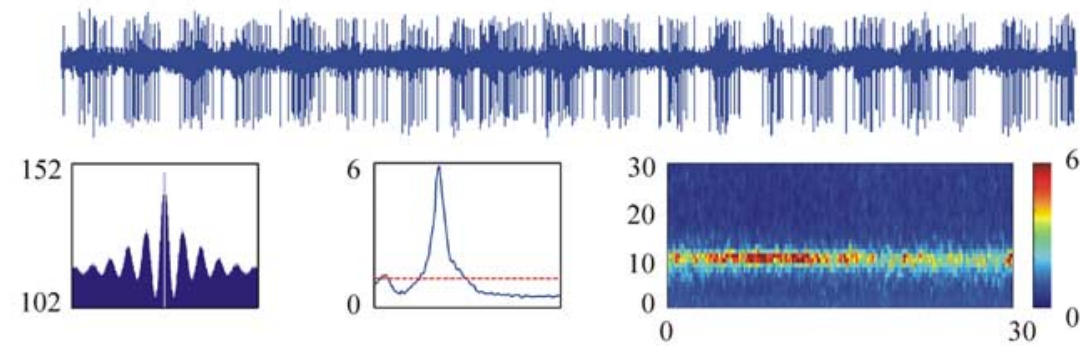

C
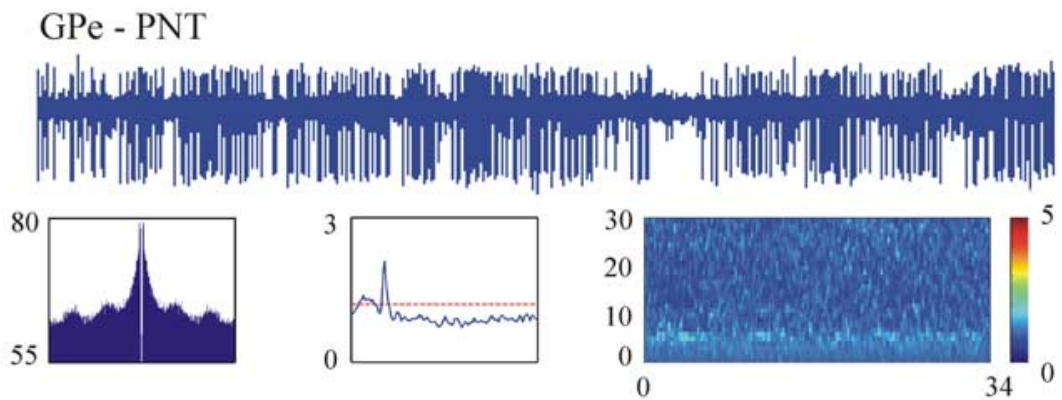

D

GPe - POT-'off'
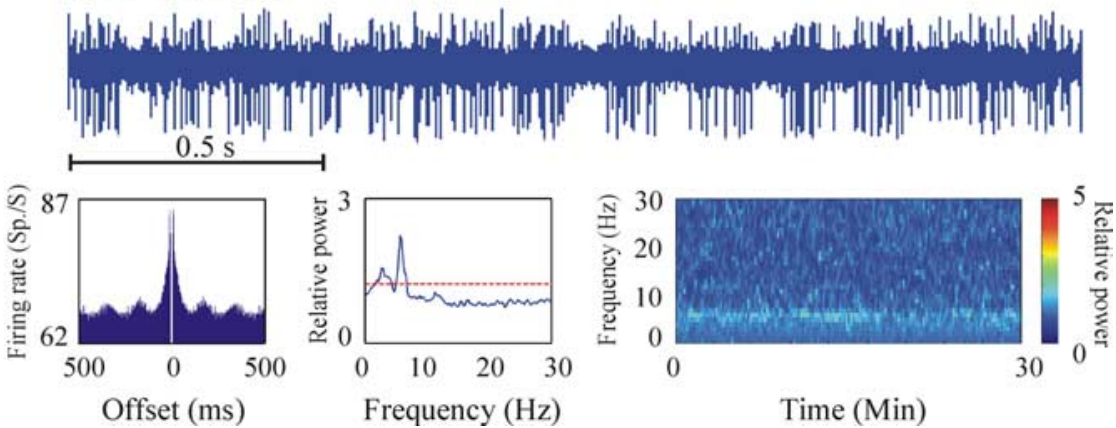

Figure 6. Examples of oscillatory activity of two GPi cells $(\boldsymbol{A}, \boldsymbol{B})$ and two GPe cells $(\boldsymbol{C}, \boldsymbol{D})$ of the vervet monkey $Q$ during the levodopa-naive parkinsonian state $(\boldsymbol{A}, \boldsymbol{C})$ and optimal treatment 0 ff state $(\boldsymbol{B}, \boldsymbol{D})$. For each unit, the top shows $2 \mathrm{~s}$ of raw analog signal. The bottom shows (from left to right) the autocorrelation, compensated (by shuffling) power spectrum, and spectrogram of the discharge of the cell. Ordinate of the autocorrelations, power spectra, and color bar of spectrograms appear at the same range for both GPi cells and both GPe cells. The examples illustrate the tendency of many GPi cells to exhibit strong $\sim 10 \mathrm{~Hz}$ oscillations compared with GPe cells that oscillate to a lesser extent and mostly at $\sim 6 \mathrm{~Hz}$. Clinical state definitions are as in Figure 3 .

GPi was more than twice that of the GPe (Fig. 5D). In the normal state, conversely, there was no significant difference between the oscillatory fractions within the GPe compared with the GPi. Examining the Off/On ratio of the fraction of oscillatory cells shows that the extent of the Off-On changes in oscillations was consistently larger in the GPi compared with the GPe. In addition, the magnitude of decrease in oscillatory percentage in response to DRT was larger in the dyskinetic treatment than in the optimal treatment in both nuclei.
The results of the cells recorded continuously over the Off-On transition are summarized in Table $4 B$. Most cells that were oscillatory in the Off state became non-oscillatory in the On state. Among all of the continuously recorded cells that were not oscillatory in the Off state, only one (a GPe cell in the dyskinetic state) became oscillatory in the On state.

The frequencies at which the pallidal cells oscillate are illustrated in Figure 8. The different frequency groups (Fig. $8 B$ ) were defined according to the clusters that are visible in Figure $8 \mathrm{~A}$. Whereas the dominant frequency of oscillations of GPe cells in both monkeys was between 4.5 and $7.5 \mathrm{~Hz}$, in GPi cells the 7.5-13.5 Hz oscillations were more than twice as common as the $4.5-7.5 \mathrm{~Hz}$ group (see also Fig. 6). The tendency of GPe and GPi cells toward 6 and $10 \mathrm{~Hz}$ oscillations, respectively, was maintained throughout all of the parkinsonian states. Of all the oscillatory (4.5-30 $\mathrm{Hz}$ ) GPe cells in monkeys Q and R, 63.4 and $70.4 \%$, respectively, only oscillated at $4.5-7.5 \mathrm{~Hz}, 22.6$ and $11.1 \%$ only oscillated at $7.5-13.5 \mathrm{~Hz}$, and 11.8 and $14.8 \%$ oscillated at both frequency domains. The mean frequency of all GPe cells that oscillated between 4.5 and $7.5 \mathrm{~Hz}$ was 5.8 and $6.1 \mathrm{~Hz}$ for monkeys $\mathrm{Q}$ and $\mathrm{R}$, respectively. Of all oscillatory $(4.5-30 \mathrm{~Hz}) \mathrm{GPi}$ cells in monkey Q, $69.5 \%$ only oscillated at $7.5-13.5 \mathrm{~Hz}, 13.4 \%$ only oscillated at $4.5-7.5 \mathrm{~Hz}$, and $13.4 \%$ oscillated at both 4.5-7.5 and 7.5-13.5 Hz. The mean frequency of all 7.5-13.5 oscillations in the GPi of monkey Q was $10.4 \mathrm{~Hz}$. The only notable difference in the frequency distribution between the different clinical states was a relatively higher fraction of 13.5-20 Hz oscillations in the GPi during On compared with Off states. Another qualitative difference between the neuronal oscillations of the GPe and GPi was the significantly ( $p<0.01$, Student's $t$ test) higher relative power of all GPi oscillations in general and particularly the $7.5-13.5 \mathrm{~Hz}$ group (Fig. 6, compare $A, B$ with $C, D$ and Fig. 8 ). The high relative power peaks at $\sim 10 \mathrm{~Hz}$ in the GPi (Fig. 8A) originated exclusively from the levodopa-naive parkinsonian state and the Off state of optimal treatment.

We failed to find neuronal oscillations at $20-30 \mathrm{~Hz}$ in the autocorrelograms of either monkey. No gamma frequency oscillations $(30-100 \mathrm{~Hz})$ were seen in monkey R. In monkey Q, a small fraction of cells oscillated at this range in the normal state (1.2 and 5.9\% of the GPe and GPi, respectively). Gamma oscillations were not detected in our spike trains in any other state, except in $2 \%$ of the GPi cells in the levodopa-naive parkinsonian state. 
Neuronal correlation

Figure 9, $A$ and $C$, illustrates oscillatory and non-oscillatory, respectively, peaked cross-correlogram and crossspectra matrices, indicating that both types of interneuronal correlation exist in the pallidum of the parkinsonian monkey. Figure 9, $B$ and $D$, which shows the flattening of the cross-correlogram and cross-spectra matrices of the same cells after administration of medication, is an example of the decreased level of neuronal oscillatory and non-oscillatory correlation in response to DRT.

Table 3, $C$ and $D$, summarizes the percentage of the correlated neuronal pairs within the GPe and GPi during each clinical state. Whereas in the normal state most neuronal pairs within the GPe of both monkeys were uncorrelated, after induction of parkinsonism, there was more than a twofold increase in the fraction of correlated pairs (Fig. 5C). Throughout the Off states of treatment, the percentage of correlated GPe pairs continued to increase, and, in the Off state of dyskinetic treatment, it was significantly higher than in the levodopa-naive parkinsonian state in both monkeys. In both monkeys, this increase in GPe correlation was attributable solely to a rise in non-oscillatory correlations. In response to DRT, the fraction of correlated GPe pairs decreased significantly in both monkeys and was not significantly different than in the normal state. Although overall the level of correlation in the GPe during the levodopanaive parkinsonian state was similar for both monkeys, its characterization varied. In the tremulous vervet monkey Q, nearly half of all significant correlations were oscillatory, whereas in the nontremulous macaque monkey $\mathrm{R}$, the oscillatory cross-correlations amounted to merely $5 \%$ of all significant correlations.

The correlation level in the normal GPi was low and nonoscillatory in nature, similar to that of the normal GPe (Fig. $5 C$ ). After induction of parkinsonism, the percentage of correlated GPi pairs increased dramatically by more than fivefold and consisted of oscillatory correlations alone. In contrast to the GPe, in the GPi there was no significant difference in the overall level of correlation between the levodopa-naive parkinsonian state and the Off states of treatment. However, like the GPe, there was a significant decrease in the relative fraction of oscillatory cross-correlations during the treatment Off states and emergence of non-oscillatory correlations that were nonexistent in the levodopa-naive parkinsonian state. Whereas in the GPe the response to DRT was similar in both optimal and dyskinetic treatment, in the GPi there was a clear difference. The decrease in correlations in the optimal On state was minimal and the correlation level remained significantly higher than in the normal state. Furthermore, all correlated pairs in this state exhibited oscillatory correlations. Conversely, in the dyskinetic On state, the GPi correlation level decreased to a near normal level and comprised nonoscillatory correlation exclusively.

Stable continuous recordings before and after the clinical in-
B
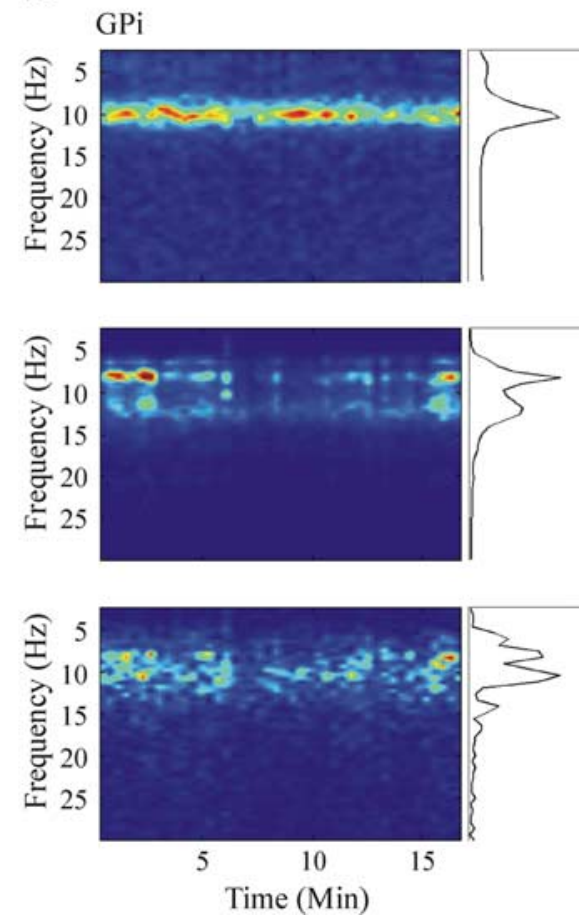

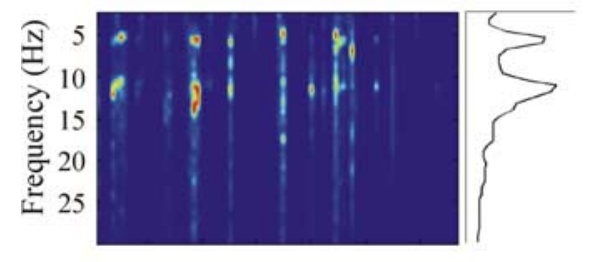

.

A

Figure 7. Examples of the complex temporal and spectral relationship between cell oscillations and tremor. $\boldsymbol{A}$ illustrates the (he same GPi cell shown in Figure $6 A$ (both are from monkey $Q$ during the PNT accelerometer, and their cross-spectrogram (from top to bottom). On the right of each panel are the corresponding auto- and fluence of DRT revealed similar effects to the population results. As with the population results, most pairs that were correlated in the Off state became uncorrelated in the On state. Only a few neuronal pairs that were not correlated in the Off state synchronized their activity after the Off-On transition (Table 4C).

The frequencies of the oscillatory cross-correlations are shown in Figure 8. In the GPi, consistent with single-cell oscillations, the main frequency was between 7.5 and $13.5 \mathrm{~Hz}$, and oscillatory cross-correlations exceeded those of the GPe in both their incidence and strength. In the GPe, conversely, the main frequency of oscillatory cross-correlations was also between 7.5 and $13.5 \mathrm{~Hz}$, in contrast to the $4.5-7.5 \mathrm{~Hz}$ oscillation that dominated the single-cell autocorrelations. Moreover, unlike the single-cell oscillations, $20-30 \mathrm{~Hz}$ oscillatory cross-correlations were identified. Higher-frequency $(>30 \mathrm{~Hz})$ oscillations were not found in our data.

\section{Discussion}

In this study, we explored the role of oscillatory and nonoscillatory pallidal activity at the level of both single-cell and interneuronal correlation, throughout the clinical states of parkinsonism and DRT. We recorded the extracellular spiking activity from the GPe (of macaque and vervet monkeys) and GPi (of the vervet monkey only). We combined tremor accelerometry, multiple-electrode recordings, population correlation, and spectral analysis (Rivlin-Etzion et al., 2006) with continuous recording of the same cells through the Off-On transition. We produced and recorded from multiple clinical states similar to those observed in parkinsonian patients (akinetic-rigid vs tremordominant PD and optimal vs dyskinetic DRT) and compared these with normal control and levodopa-naive parkinsonian 
A
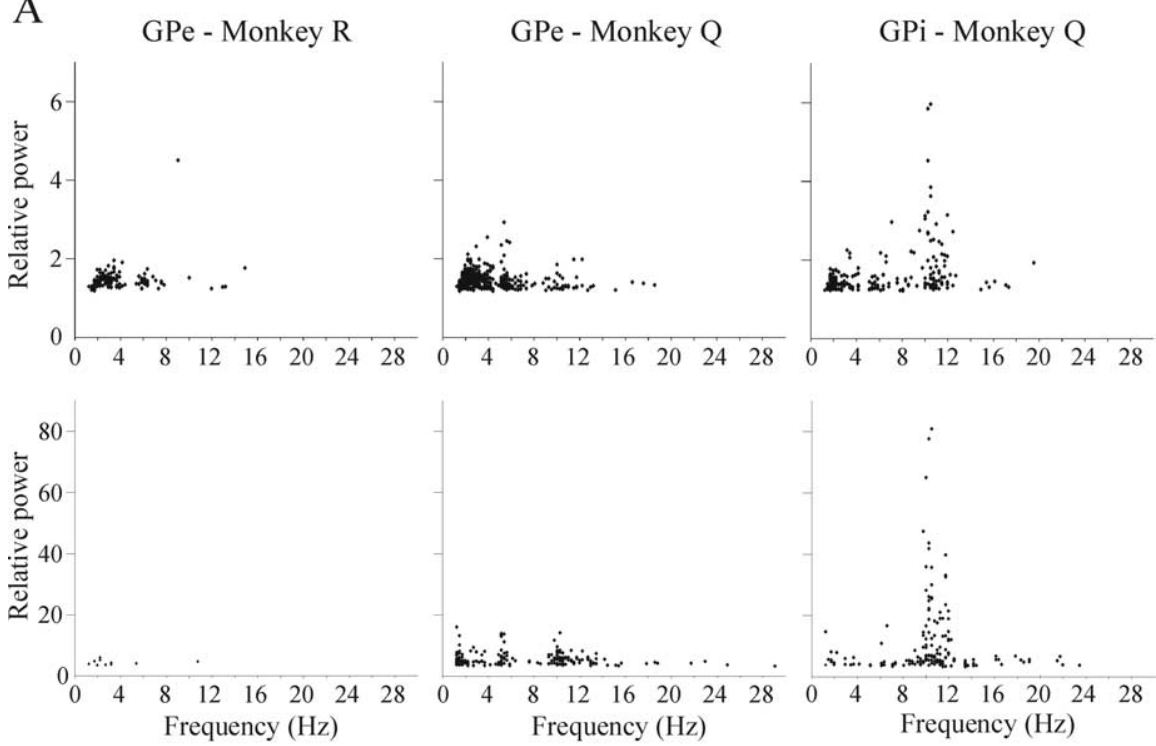

B

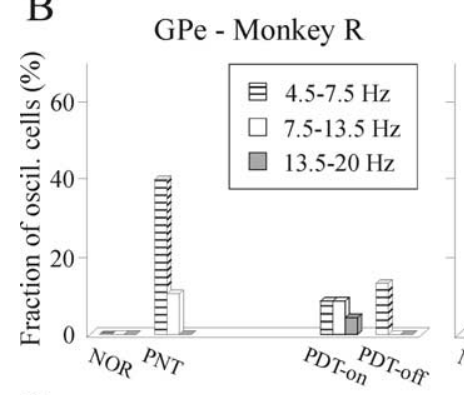

$$
\text { GPe - Monkey Q }
$$
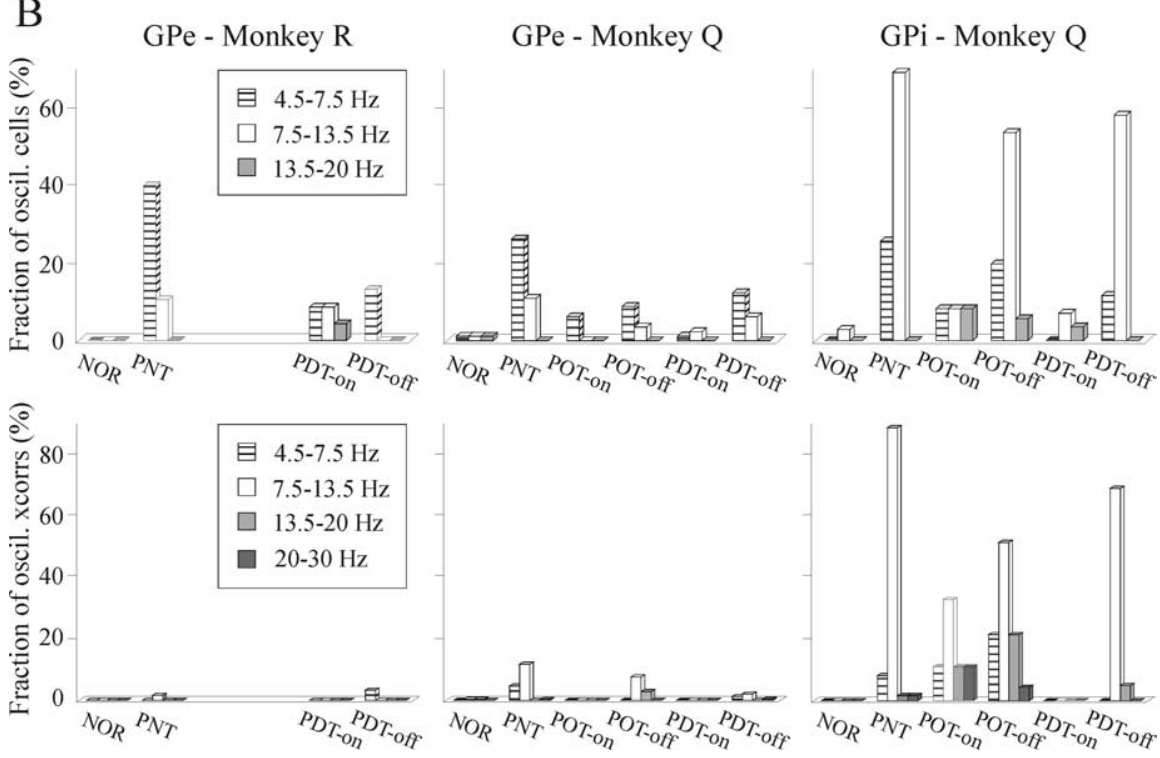

Figure 8. Relative power and frequency distribution of single-cell oscillations and pairwise oscillatory correlations. $A$, Plot of the relative power of the power spectra peaks of single-cell oscillations (top row) and pairwise oscillatory correlations (bottom row) as a function of their frequency. All significant $1-30 \mathrm{~Hz}$ peaks (possibly more than 1 peak for a single cell or a single neuronal pair) from all clinical states were included. The plot demonstrates the different frequency clusters, the higher relative power of GPi oscillations, and the appearance of the $20-30 \mathrm{~Hz}$ oscillatory correlations compared with the absence of such frequencies in the single-cell oscillations. $\boldsymbol{B}$, Frequency distribution of single-cell oscillations (top row) and oscillatory pairwise correlations (bottom row). The different frequency groups were defined according to the clusters seen in $\boldsymbol{A}$. Units or neuronal pairs that oscillated in more than one frequency group were counted more than once. Despite the different dominant frequencies of single-cell oscillations within the GPe and GPi $(\sim 6$ and $\sim 10 \mathrm{~Hz}$, respectively), in both nuclei the chief frequency of oscillatory correlations is $\sim 10$ $\mathrm{Hz}$. Clinical state definitions are as in Figure 3.

states, which are lacking in human studies. Our fast-induction MPTP model might not exactly reflect the slow and progressive anatomical/biochemical compensatory changes in the basal ganglia that occur in idiopathic PD or in the slowprogressive MPTP models (Russ et al., 1991; Perez et al., 1994). However, it enabled prolonged recording over the multiple PD states in the same animal, including the state of levodopa-induced dyskinesia. Using these methods, we demonstrate that the tremor characteristics after exposure to DRT do not resemble those of the normal or the levodopa-naive state. Moreover, the balance between the neural activity (rate, pattern, and pairwise correlations) between the GPe and GPi is disrupted after MPTP treatment. These results therefore call for reappraisal of our current models of basal ganglia and Parkinson's disease pathophysiology (see below).

\section{Tremor analysis}

The tremor analysis confirms previous reports of an abundance of tremor episodes in the MPTP-treated vervet monkey compared with the relatively nontremulous macaque (Redmond et al., 1985; Bergman et al., 1994). In both monkeys, there was either no change or even an increase in the extent of the tremor in response to DRT. This phenomenon is occasionally observed in human patients (Vidailhet et al., 1999) and may be attributed to tremor masking by severe akinesia and rigidity. As in human studies (Hurtado et al., 2000; Raethjen et al., 2000; Ben-Pazi et al., 2001), the DRT states were characterized by a low coherence level between the tremor of the limbs even in Off states; however, in the levodopa-naive state, limb tremor was highly coherent. These results indicate (in line with the electrophysiological studies discussed below) that DRT causes major changes in the functional organization of the basal ganglia. The Off state of human parkinsonian patients, after many years of DRT, therefore may not represent a pure dopamine-depleted state; rather, it may be the result of complex interactions between natural compensatory processes for dopamine depletion, as well as the neuronal responses to chronic DRT.

\section{Neuronal firing rates}

In the analysis of neuronal firing rates in the levodopa-naive parkinsonian state, we only found a significant change in the discharge rate of GPe cells in the vervet monkey. These inconclusive results are in line with other primate studies (Boraud et al., 2002). Nevertheless, as in primate (Filion et al., 1991; Papa et al., 1999; Boraud et al., 2001) and human (Levy et al., 2001; Stefani et al., 2002) studies, the increase in GPe and decrease in GPi rates in response to DRT were robust. As a result, during the Off periods, GPi rates were significantly higher than the GPe rates, whereas the opposite occurred in On periods.

\section{Single-cell oscillations}

The low fraction of oscillatory cells in both nuclei significantly increased after induction of parkinsonism, and different degrees of decrease were observed in response to DRT. Nevertheless, notable differences were found between the neuronal oscillations of the two pallidal nuclei. In the GPi, the high oscillatory level was maintained throughout the DRT-Off states, whereas in the GPe 
of both monkeys, it was significantly lower during the DRT-Off states compared with the levodopa-naive parkinsonian state. Throughout all MPTP and DRT states, the fraction and relative power of oscillatory cells within the GPi was higher than that of the GPe, and the dominant frequency of single-cell oscillations in the GPi was 7.513.5 $\mathrm{Hz}$ compared with $4.5-7.5 \mathrm{~Hz}$ in the GPe. The small fraction of cells oscillating in more than one frequency and the uneven distribution of the two main frequencies between the GPe and GPi may indicate that each frequency results from genuine physiological characteristics of the cells or the network rather than from a harmonic artifact of the spectral analysis.

Human studies have reported a high fraction of GPi cells oscillating at the tremor frequency (Hutchinson et al., 1997b); however, recent primate (Raz et al., 2000) and human (Hurtado et al., 1999, 2005; Lemstra et al., 1999) studies show that these oscillations are not fully coherent with the simultaneous recorded tremor. Our results also reveal several discrepancies between the pallidal oscillations and the tremor. Moreover, comparison of the GPe activity in the tremulous and nontremulous animals implies that the formation of oscillatory correlations rather than single-cell oscillations plays a major role in tremor generation. We demonstrate that, in contrast to similar fractions of oscillatory cells and correlated pairs in the GPe of both animals in the levodopa-naive state, most pairwise correlations in the nontremulous macaque are non-oscillatory, whereas in the tremulous vervet, nearly half are oscillatory. However, these findings are population based and circumstantial; therefore, additional studies are needed to evaluate the specific temporal relationships between single or assembly neuronal oscillations and the tremor phenomenon.

\section{Interneuronal correlations}

In line with previous MPTP studies (Nini et al., 1995; Raz et al., 2000), we found an increased level of pairwise neuronal correlations in both pallidal segments after MPTP treatment. This abnormal synchronization decreased by a variable extent in response to DRT. The disparity between the two pallidal nuclei was also present in terms of interneuronal correlations. In contrast to the similarly low interneuronal synchronization in both GPe and GPi in the normal state, after MPTP, the level of neuronal synchronization in the GPi significantly exceeded that of the GPe. Moreover, whereas neuronal correlations in the GPe were mostly non-oscillatory, the vast majority of correlated GPi pairs exhibited $\sim 10 \mathrm{~Hz}$ oscillatory correlations.

A recent study in human patients only found oscillatory single-cell activity and interneuronal synchronization in tremulous patients and failed to find any non-oscillatory correlations (Levy et al., 2002). These results may imply that the oscillatory
B

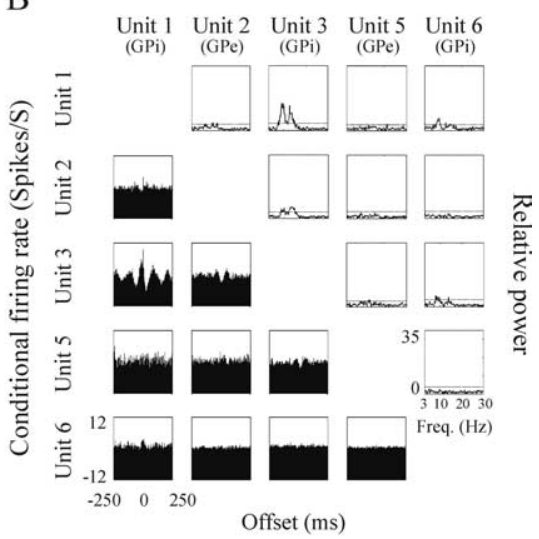

$\mathrm{D}$

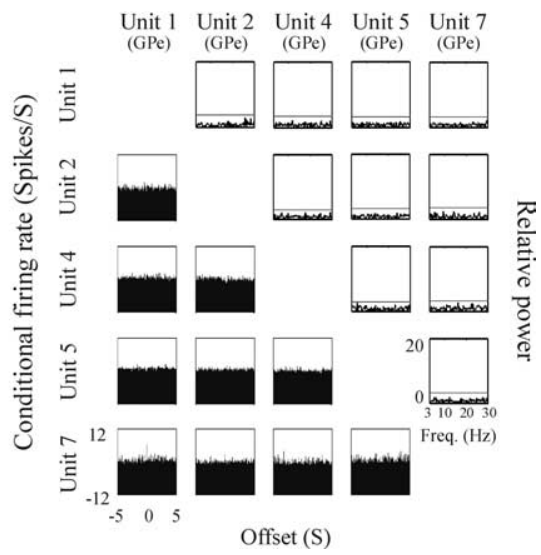

Figure 9. Example of the decrease in neuronal synchronization in response to DRT. In each panel, the left bottom triangle is the cross-correlogram matrix, and the right top triangle is the cross-spectra matrix. $A, B$, Prominent oscillatory synchronization in the riod of optimal treatment state $(\boldsymbol{A})$ is significantly decreased after response to the medication $(\boldsymbol{B})$. The matrices were subsequent 0 n period 22 min later. $\boldsymbol{C}, \boldsymbol{D}$, Wide peaks of non-oscillatory synchronization in the Off period ( $\boldsymbol{C}$ of dyskinetic treatment recordings of the same five GPe cells shown in Figure 4, during the Off period and the subsequent On period 26 min later. The ordinate of the conditional firing rate is expressed in the same range of \pm 12 spikes/s around the average firing rate. The ordinate of the relative power is also the same for all cross-spectra in $\boldsymbol{A}$ and $\boldsymbol{B}(0-35 \mathrm{~Hz})$ and for all cross-spectra in $\boldsymbol{C}$ and $\boldsymbol{D}(0-20 \mathrm{~Hz})$.

correlation is merely a byproduct of the tremor or independent oscillators with similar frequencies. We found a significant fraction of non-oscillatory synchronization in our recordings. Additional findings presented here indicating different frequency regimens for the autocorrelation and cross-correlation functions, as well as distinct modulation of the tremor, single-cell, and interneuronal oscillations by the DRT further suggest that the increased neuronal synchronization within the pallidum is not simply a reflection of tremor or single-cell oscillations.

\section{Neuronal substrates of levodopa-induced dyskinesia}

A major aim of our study was to characterize the physiological differences in neuronal activity during optimal and dyskinetic treatment. Our results are in line with a previous study that found lower neuronal rates in the GPi during dyskinetic On compared with optimal On (Papa et al., 1999). In addition, we show that the imbalance of GPe/GPi firing rates in response to DRT is further increased in the dyskinetic On state. We found a more pronounced Off-On decrease in oscillatory activity in the dyskinetic state in both pallidal nuclei. Similarly, the relative proportion of oscillatory correlations was lower in the dyskinetic On state. After 
introduction of DRT, prolonged synchronous bursts of GPe neurons appeared in the Off states and were considerably more prominent after the development of dyskinesia. We propose that the weakening of network oscillations within the GPi in the dyskinetic state enables the emerging of bursting activity in the GPe or other structures such as the thalamus to manifest in the form of dyskinesia. The occurrence of dyskinesia during On rather than Off periods can be attributed to the concurrent decrease of GPi rates, which further reduces its inhibitory effect.

We conclude that dopamine depletion and replacement therapy result in changes in many aspects of neural activity (e.g., rate, pattern, and synchronization) over the whole basal ganglia cortical networks; consequently, the GPe/GPi balance that exists in the normal state (in these three aspects of neuronal activity) is disturbed and fails to reinstate thereafter. Recent studies (Bolam et al., 2000; Lee et al., 2004; Levesque and Parent, 2005) have indicated that the GPe should not be considered as a simple relay structure in the indirect striatopallidal pathway. Rather, the GPe plays a major role in the control of the entire basal ganglia circuitry. Nevertheless, despite the strong inhibitory projections from the GPe to the GPi (Hazrati et al., 1990; Bolam et al., 2000), the GPe activity does not impose mirror changes in the GPi neuronal activity. Our results, demonstrating disruption of the GPe/ GPi balance after MPTP and DRT, suggest that this imbalance is a hallmark of the pathophysiology of parkinsonism and DRT. They further indicate that chronic DRT may cause additional abnormal organization of the basal ganglia networks beyond the effects of dopamine depletion. Future attempts at improving invasive therapies of human patients with advanced PD and DRT side effects should therefore aim at restoring the full spectrum of normal GPe and GPi activity.

\section{References}

Albin RL, Young AB, Penney JB (1989) The functional anatomy of basal ganglia disorders. Trends Neurosci 12:366-375.

Aziz TZ, Peggs D, Sambrook MA, Crossman AR (1991) Lesion of the subthalamic nucleus for the alleviation of 1-methyl-4-phenyl-1,2,3,6tetrahydropyridine (MPTP)-induced parkinsonism in the primate. Mov Disord 6:288-293.

Bar-Gad I, Ritov Y, Bergman H (2001) Failure in identification of overlapping spikes from multiple neuron activity causes artificial correlations. J Neurosci Methods 107:1-13.

Ben-Pazi H, Bergman H, Goldber JA, Giladi N, Hansel D, Reches A, Simon ES (2001) Synchrony of rest tremor in multiple limbs in parkinson's disease: evidence for multiple oscillators. J Neural Transm 108:287-296.

Bergman H, Wichmann T, DeLong MR (1990) Reversal of experimental parkinsonism by lesions of the subthalamic nucleus. Science 249:1436-1438.

Bergman H, Wichmann T, Karmon B, DeLong MR (1994) The primate subthalamic nucleus. II. Neuronal activity in the MPTP model of parkinsonism. J Neurophysiol 72:507-520.

Bergman H, Feingold A, Nini A, Raz A, Slovin H, Abeles M, Vaadia E (1998) Physiological aspects of information processing in the basal ganglia of normal and parkinsonian primates. Trends Neurosci 21:32-38.

Bloomfield P (1976) Fourier analysis of time series: an introduction. New York: Wiley.

Bolam JP, Hanley JJ, Booth PA, Bevan MD (2000) Synaptic organisation of the basal ganglia. J Anat 196:527-542.

Boraud T, Bezard E, Guehl D, Bioulac B, Gross C (1998) Effects of L-DOPA on neuronal activity of the globus pallidus externalis (GPe) and globus pallidus internalis (GPi) in the MPTP-treated monkey. Brain Res 787:157-160.

Boraud T, Bezard E, Bioulac B, Gross CE (2001) Dopamine agonist-induced dyskinesias are correlated to both firing pattern and frequency alterations of pallidal neurones in the MPTP-treated monkey. Brain 124:546-557.

Boraud T, Bezard E, Bioulac B, Gross CE (2002) From single extracellular unit recording in experimental and human Parkinsonism to the develop- ment of a functional concept of the role played by the basal ganglia in motor control. Prog Neurobiol 66:265-283.

Brillinger DR (1981) Time series-data analysis and theory. San Francisco: Holden-Day.

Contreras CM, Mexicano G, Guzman-Flores CA (1981) Stereotaxic brain atlas of the green monkey (Cercopithecus Aethiops Aethiops). Bol Est Med Biol 31:383-428.

DeLong MR (1990) Primate models of movement disorders of basal ganglia origin. Trends Neurosci 13:281-285.

Doudet DJ, Gross C, Arluison M, Bioulac B (1990) Modifications of precentral cortex discharge and EMG activity in monkeys with MPTP-induced lesions of DA nigral neurons. Exp Brain Res 80:177-188.

Filion M, Tremblay L (1991) Abnormal spontaneous activity of globus pallidus neurons in monkeys with MPTP-induced parkinsonism. Brain Res 547:142-151.

Filion M, Tremblay L, Bedard PJ (1991) Effects of dopamine agonists on the spontaneous activity of globus pallidus neurons in monkeys with MPTPinduced parkinsonism. Brain Res 547:152-161.

Goldberg JA, Boraud T, Maraton S, Haber SN, Vaadia E, Bergman H (2002) Enhanced synchrony among primary motor cortex neurons in the 1-methyl-4-phenyl-1,2,3,6-tetrahydropyridine primate model of Parkinson's disease. J Neurosci 22:4639-4653.

Hazrati LN, Parent A, Mitchell S, Haber SN (1990) Evidence for interconnections between the two segments of the globus pallidus in primates: a PHA-L anterograde tracing study. Brain Res 533:171-175.

Heimer G, Bar-Gad I, Goldberg JA, Bergman H (2002) Dopamine replacement therapy reverses abnormal synchronization of pallidal neurons in the 1-methyl-4-phenyl-1,2,3,6-tetrahydropyridine primate model of parkinsonism. J Neurosci 22:7850-7855.

Hoehn MM, Yahr MD (1967) Parkinsonism: onset, progression and mortality. Neurology 17:427-442.

Hurtado JM, Gray CM, Tamas LB, Sigvardt KA (1999) Dynamics of tremorrelated oscillations in the human globus pallidus: a single case study. Proc Natl Acad Sci USA 96:1674-1679.

Hurtado JM, Lachaux JP, Beckley DJ, Gray CM, Sigvardt KA (2000) Interand intralimb oscillator coupling in parkinsonian tremor. Mov Disord 15:683-691.

Hurtado JM, Rubchinsky LL, Sigvardt KA, Wheelock VL, Pappas CT (2005) Temporal evolution of oscillations and synchrony in GPi/muscle pairs in Parkinson's disease. J Neurophysiol 93:1569-1584.

Hutchinson WD, Levy R, Dostrovsky JO, Lozano AM, Lang AE (1997a) Effects of apomorphine on globus pallidus neurons in parkinsonian patients. Ann Neurol 42:767-775.

Hutchinson WD, Lozano AM, Tasker RR, Lang AE, Dostrovsky JO (1997b) Identification and characterization of neurons with tremor- frequency activity in human globus pallidus. Exp Brain Res 113:557-563.

Imbert C, Bezard E, Guitraud S, Boraud T, Gross CE (2000) Comparison of eight clinical rating scales used for the assessment of MPTP-induced parkinsonism in the Macaque monkey. J Neurosci Methods 96:71-76.

Krack P, Batir A, Van Blercom N, Chabardes S, Fraix V, Ardouin C, Koudsie A, Limousin PD, Benazzouz A, Lebas JF, Benabid AL, Pollak P (2003) Five-year follow-up of bilateral stimulation of the subthalamic nucleus in advanced Parkinson's disease. N Engl J Med 349:1925-1934.

Kumar R, Lang AE, Rodriguez-Oroz MC, Lozano AM, Limousin P, Pollak P, Benabid AL, Guridi J, Ramos E, van der Linden C, Vandewalle A, Caemaert J, Lannoo E, van den Abbeele D, Vingerhoets G, Wolters M, Obeso JA (2000) Deep brain stimulation of the globus pallidus pars interna in advanced Parkinson's disease. Neurology 55:S34-S39.

Lang AE, Lozano AM, Montgomery E, Duff J, Tasker R, Hutchinson W (1997) Posteroventral medial pallidotomy in advanced Parkinson's disease. N Engl J Med 337:1036-1042.

Lee CR, Abercrombie ED, Tepper JM (2004) Pallidal control of substantia nigra dopaminergic neuron firing pattern and its relation to extracellular neostriatal dopamine levels. Neuroscience 129:481-489.

Lemstra AW, Verhagen ML, Lee JI, Dougherty PM, Lenz FA (1999) Tremor-frequency $(3-6 \mathrm{~Hz})$ activity in the sensorimotor arm representation of the internal segment of the globus pallidus in patients with Parkinson's disease. Neurosci Lett 267:129-132.

Levesque M, Parent A (2005) The striatofugal fiber system in primates: a reevaluation of its organization based on single-axon tracing studies. Proc Natl Acad Sci USA 102:11888-11893.

Levy R, Hazrati LN, Herrero MT, Vila M, Hassani OK, Mouroux M, Ruberg 
M, ASENSI H, Agid Y, Feger J, Obeso JA, Parent A, Hirsch EC (1997) Re-evaluation of the functional anatomy of the basal ganglia in normal and Parkinsonian states. Neuroscience 76:335-343.

Levy R, Hutchison WD, Lozano AM, Dostrovsky JO (2000) High-frequency synchronization of neuronal activity in the subthalamic nucleus of parkinsonian patients with limb tremor. J Neurosci 20:7766-7775.

Levy R, Dostrovsky JO, Lang AE, Sime E, Hutchison WD, Lozano AM (2001) Effects of apomorphine on subthalamic nucleus and globus pallidus internus neurons in patients with Parkinson's disease. J Neurophysiol $86: 249-260$.

Levy R, Hutchison WD, Lozano AM, Dostrovsky JO (2002) Synchronized neuronal discharge in the basal ganglia of parkinsonian patients is limited to oscillatory activity. J Neurosci 22:2855-2861.

Marsden CD, Obeso JA (1994) The functions of the basal ganglia and the paradox of stereotaxic surgery in Parkinson's disease. Brain 117:877-897.

Merello M, Balej J, Delfino M, Cammarota A, Betti O, Leiguarda R (1999) Apomorphine induces changes in GPi spontaneous outflow in patients with Parkinson's disease. Mov Disord 14:45-49.

Miller WC, DeLong MR (1987) Altered tonic activity of neurons in the globus pallidus and subthalamic nucleus in the primate MPTP model of parkinsonism. In: The basal ganglia II (Carpenter MB, Jayaraman A, eds), pp 415-427. New York: Plenum.

Nini A, Feingold A, Slovin H, Bergman H (1995) Neurons in the globus pallidus do not show correlated activity in the normal monkey, but phaselocked oscillations appear in the MPTP model of parkinsonism. J Neurophysiol 74:1800-1805.

Papa SM, DeSimone R, Fiorani M, Oldfield EH (1999) Internal globus pallidus discharge is nearly suppressed during levodopa-induced dyskinesias. Ann Neurol 46:732-738.

Paxinos G, Huang X-F, Toga AW (2000) The rhesus monkey brain in stereotaxic coordinates. San Diego: Academic.

Perez OI, Oset C, Luquin MR, Herrero MT, Obeso JA, Del Rio J (1994) MPTP-induced parkinsonism in primates: pattern of striatal dopamine loss following acute and chronic administration. Neurosci Lett 175:121-125.

Pessiglione M, Guehl D, Rolland AS, Francois C, Hirsch EC, Feger J, Tremblay L (2005) Thalamic neuronal activity in dopamine-depleted primates: evidence for a loss of functional segregation within basal ganglia circuits. J Neurosci 25:1523-1531.

Raethjen J, Lindemann M, Schmaljohann H, Wenzelburger R, Pfister G, Deuschl G (2000) Multiple oscillators are causing parkinsonian and essential tremor. Mov Disord 15:84-94.

Raz A, Vaadia E, Bergman H (2000) Firing patterns and correlations of spontaneous discharge of pallidal neurons in the normal and the tremulous 1-methyl-4-phenyl-1,2,3,6-tetrahydropyridine vervet model of parkinsonism. J Neurosci 20:8559-8571.

Redmond DE, Roth RH, Sladek JR (1985) MPTP produces classic parkinsonian syndrome in African green monkeys. Soc Neurosci Abstr 11:54.1.

Rivlin-Etzion M, Ritov Y, Heimer G, Bergman H, Bar-Gad I (2006) Local shuffling of spike trains boosts the accuracy of spike train spectral analysis. J Neurophysiol 95:3245-3256.

Russ H, Mihatsch W, Gerlach M, Riederer P, Przuntek H (1991) Neurochemical and behavioural features induced by chronic low dose treatment with 1-methyl-4-phenyl-1,2,3,6-tetrahydropyridine (MPTP) in the common marmoset: implications for Parkinson's disease? Neurosci Lett 123:115-118.

Song DD, Haber SN (2000) Striatal responses to partial dopaminergic lesion: evidence for compensatory sprouting. J Neurosci 20:5102-5114.

Stefani A, Bassi A, Mazzone P, Pierantozzi M, Gattoni G, Altibrandi MG, Giacomini P, Peppe A, Bernardi G, Stanzione P (2002) Subdyskinetic apomorphine responses in globus pallidus and subthalamus of parkinsonian patients: lack of clear evidence for the "indirect pathway." Clin Neurophysiol 113:91-100.

Vidailhet M, Bonnet AM, Marconi R, Durif F, Agid Y (1999) The phenomenology of L-dopa-induced dyskinesias in Parkinson's disease. Mov Disord 14 [Suppl 1]:13-18.

Walter BL, Vitek JL (2004) Surgical treatment for Parkinson's disease. Lancet Neurol 3:719-728.

Watts RL, Mandir AS (1992) The role of motor cortex in the pathophysiology of voluntary movement deficits associated with parkinsonism. Neurol Clin 10:451-469.

Wichmann T, Soares J (2006) Neuronal firing before and after burst discharges in the monkey basal ganglia is predictably patterned in the normal state and altered in parkinsonism. J Neurophysiol 95:2120-2133. 